\title{
Existence and Stability of Travelling Front Solutions for General Auto-catalytic Chemical Reaction Systems
}

\author{
Y. $\mathrm{Li}^{1}, \mathrm{Y} . \mathrm{Wu}^{2} *$ \\ ${ }^{1}$ Department of Mathematics, Xi'an Jiaotong University, Xi'an 710049, P.R. China \\ and Department of Mathematics and Statistics, Wright State University, \\ Dayton, OH45435, USA \\ ${ }^{2}$ College of Mathematical Sciences, Capital Normal University, \\ Beijing, 100048, P.R. China
}

\begin{abstract}
This paper is concerned with the existence and stability of travelling front solutions for more general autocatalytic chemical reaction systems $u_{t}=d u_{x x}-u f(v), \quad v_{t}=v_{x x}+u f(v)$ with $d>0$ and $d \neq 1$, where $f(v)$ has super-linear or linear degeneracy at $v=0$. By applying Lyapunov-Schmidt decomposition method in some appropriate exponentially weighted spaces, we obtain the existence and continuous dependence of wave fronts with some critical speeds and with exponential spatial decay for $d$ near 1. By applying special phase plane analysis and approximate center manifold theorem, the existence of traveling waves with algebraic spatial decay or with some lower exponential decay is also obtained for $d>0$. Further, by spectral estimates and Evans function method, the wave fronts with exponential spatial decay are proved to be spectrally or linearly stable in some suitable exponentially weighted spaces. Finally, by adopting the main idea of proof in [12] and some similar arguments as in [21], the waves with critical speeds or with non-critical speeds are proved to be locally exponentially stable in some exponentially weighted spaces and Lyapunov stable in $C_{\text {unif }}(\mathbb{R})$ space, if the initial perturbation of the waves is small in both the weighted and unweighted norms; the perturbation of the waves also stays small in $L_{1}(\mathbb{R})$ norm and decays algebraically in $C_{\text {unif }}(\mathbb{R})$ norm, if the initial perturbation is in addition small in $L_{1}$ norm.
\end{abstract}

Keywords and phrases: travelling waves, existence, stability, spectral analysis

Mathematics Subject Classification: 35B35, 35K55, 35A18, 35L67, 35L65

\section{Introduction and statement of main results}

In this paper we investigate the following reaction-diffusion systems

$$
\left\{\begin{array}{l}
u_{t}=d u_{x x}-u f(v), \\
v_{t}=v_{x x}+u f(v),
\end{array} \quad x \in \mathbb{R}, t>0 ;\right.
$$

*Corresponding author. E-mail: yaping_wu@hotmail.com 
with $f(v)$ satisfying

$$
f(0)=0, \quad f(v)>0, v \in(0,1] .
$$

System (1.1) has been derived to describe the autocatalytic step in isothermal, autocatalytic chemical reaction scheme, where $u$ and $v$ are the concentrations of reactant A and autocatalyst B. System (1.1) with $f(v)=v^{p}$ can describe the autocatalytic chemical reaction with $p$ order reaction rate:

$$
A+p B \rightarrow(p+1) B, \text { with rate } k u v^{p} .
$$

For the case $f(v)=k_{1} v^{m}+k_{2} v^{n}$ with positive $k_{1}, k_{2}, m$ and $n$, system (1.1) describes some mixed order autocatalytic chemical reaction. System (1.1) can also describe thermal-diffusive combustion problems or some epidemic models in mathematical biology.

In this paper we are only interested in the existence and stability of travelling wave solutions $\left(U_{c}(x-\right.$ $\left.c t), V_{c}(x-c t)\right)$ of $(1.1)$ connecting $(0,1)$ and $(1,0)$, which correspond to chemical wavefronts, flame wavefronts or epidemic wavefronts, with $\left(U_{c}(z), V_{c}(z)\right)$ satisfying the following system

$$
\left\{\begin{array}{l}
d U_{c}^{\prime \prime}(z)+c U_{c}^{\prime}(z)-U_{c} f\left(V_{c}\right)=0, \quad z \in \mathbb{R}, \\
V_{c}^{\prime \prime}(z)+c V_{c}^{\prime}(z)+U_{c} f\left(V_{c}\right)=0, \quad z \in \mathbb{R}, \\
\left(U_{c}(-\infty), V(-\infty)\right)=(0,1), \quad\left(U_{c}(+\infty), V_{c}(+\infty)\right)=(1,0) .
\end{array}\right.
$$

We first state some well-known results on the existence of travelling wave $\left(U_{c}(z), V_{c}(z)\right)$ of $(1.3)$. For the case $d=1$, it is easy to see that

$$
U_{c}(z)+V_{c}(z) \equiv 1
$$

with $V_{c}(z)$ satisfying

$$
V_{z z}+c V_{z}+(1-V) f(V)=0, \quad z \in \mathbb{R}
$$

and

$$
V(-\infty)=1, \quad V(+\infty)=0 .
$$

For the case $f(v)=v$, equation (1.5) becomes Fisher equation, it is well known that there exists a travelling front solution $V_{c}(x-c t)$ to Fisher equation satisfying (1.6) if and only if $c \geq 2$. It is also well known that the wave $V_{c}(z)$ with the minimal speed $c=2$ decays to zero with the rate $z e^{-z}$, while the wave with a noncritical speed $c>2$ decays to zero with a slower exponential rate $e^{\left(-c+\sqrt{c^{2}-4}\right) z / 2}$. When the initial value decays exponentially at $z=+\infty$, the stability of travelling front solution with the critical speed or a noncritical speed for Fisher equation is also investigated in $[7,18,24,28]$, where it was shown that the exponential decaying rate of the initial value determines the convergence of the solution. For more general initial value with non-exponentially spatial decay, the large time behavior of solution to Fisher equation has been recently investigated in [13].

For more general $f(v)$ satisfying

$$
f \in C^{2}([0,1]), \quad f(0)=0, f^{\prime}(0)>0 \text { and } f(v)>0 \text { for } v \in(0,1],
$$

equation (1.5) is called a generalized Fisher equation or KPP equation. It is well-known that there exists a minimal speed $c_{*}^{0} \in\left[2 \sqrt{f^{\prime}(0)}, \sup _{v \in(0,1)} \sqrt{f(v) / v}\right]$, such that (1.5) has a traveling front solution $V(x-c t)$ if and only if $c \geq c_{*}^{0}$. In addition, the traveling front solution with noncritical speed $c>c_{*}^{0}$ is locally exponentially stable in some exponentially weighted spaces.

For the case $f(v)=v^{p}$ with $p>1$, equation (1.5) is also called $p$-degree Fisher equation. It has been proved in [6] and [26] that there exists a minimal speed $c_{*}^{0}(p)>0$ such that there exists a travelling wave $V_{c}(z)$ of (1.5)-(1.6) if and only if $c \geq c_{*}^{0}(p)$, and $V_{c}(z)$ satisfies $V_{c}^{\prime}(z)<0$ for $z \in R$ and

$$
\left\{\begin{array}{l}
V_{c}(z) \sim z^{-\frac{1}{p-1}} \quad \text { as } z \rightarrow+\infty, \\
1-V_{c}(z) \sim \mathrm{e}^{\sigma_{c}^{-} z} \text { as } z \rightarrow-\infty,
\end{array} \text { if } c>c_{*}^{0}(p) ;\right.
$$




$$
\left\{\begin{array}{l}
V_{c}(z) \sim \mathrm{e}^{-c z} \quad \text { as } z \rightarrow+\infty, \\
1-V_{c}(z) \sim \mathrm{e}^{\sigma_{c}^{-} z} \text { as } z \rightarrow-\infty,
\end{array} \text { if } c=c_{*}^{0}(p) .\right.
$$

The upper and lower bounds of the critical speed $c_{*}^{0}(p)$ were investigated in [22] and [10].

By applying similar phase plane analysis as in [6] and applying center manifold theorems, the above mentioned existence results for $p$ degree Fisher equations are still valid for equation (1.5) with more general $f(v)$ satisfying

$$
f \in C^{2}((0,1]), f(0)=0, f(v)>0 \text { for } v \in(0,1], \text { and } \lim _{v \rightarrow 0^{+}} \frac{f(v)}{v^{p}}=k_{0}>0 \text { for some } p>1,
$$

i.e. there exists a minimal speed $c_{*}^{0}>0$ such that there exists a traveling wave $V_{c}(x-c t)$ satisfying $(1.6)$ if and only if $c \geq c_{*}^{0}$. In addition, $V_{c}(z)$ satisfies (1.8) or (1.9).

The local asymptotic stability of travelling front solutions of $p$-degree Fisher equation with noncritical speeds or with the critical speed in some exponentially weighted spaces or in some polynomially weighed spaces have been obtained in [16][32] and [30], the global exponential stability of the wave front with the critical speed is also obtained in [30]. The stability results obtained in [30,32] also imply that the solution for the $p$ degree Fisher equation tends to the wave with the critical speed, if the initial value decays to zero exponentially or algebraically with some higher algebraic rate at $z=\infty$; however, it tends to the wave with some noncritical speed and always decays to zero at $z=\infty$ with the same algebraic rate at any time, if the initial value is close to a wave with some noncritical speed and decays to zero algebraically at $z=\infty$ with the same algebraic rate. For higher dimension space, the existence and stability of travelling waves for $p$-degree Fisher equation is investigated in [4].

For $d=0$ and $f(v)=v^{p}$ with $p>1$, the existence of the traveling front solutions for system (1.3) as well as the estimates of the minimal speed $c_{\min }(p)$ are obtained in $[15,20,27]$ by applying phase plane analysis. Besides, it is also shown in [15] that the wave with the minimal speed decays to $(1,0)$ exponentially as $z \rightarrow \infty$, and those with noncritical speeds decay to $(1,0)$ algebraically at $z=\infty$. The numerical results and some theoretical analysis in $[3,20]$ show that when $d=0$ or $d$ is near zero and $p$ is large enough the traveling front solutions to system (1.3) become unstable and there exist some oscillatory waves which may be stable.

For $d>0, d \neq 1$ and $f(v)=v+k v^{2}$, the existence and stability of traveling front solutions of the autocatalytic system (1.1) was investigated in [11] for all $k \geq 0$. If $d \leq 1$, it was proved in [11] that there exists a minimal speed $c_{*}(d, k) \geq 2$ such that system (1.1) has a traveling wave if and only if $c \geq c_{*}(d, k)$. Moreover, there exists a constant $k_{*}(d) \in[(3 d-1) /(3 d-2), 2]$ such that the minimal speed $c_{*}(d, k)$ satisfies

$$
c_{*}(d, k)=2 \text { if } k \leq k^{*}(d) \text { and } c_{*}(d, k)>2 \text { if } k>k_{*}(d) .
$$

By applying energy method, the wave fronts with speed $c \geq 2$ were proved to be locally asymptotically stable in some exponentially weighted spaces when $d$ is near 1 and $k \geq 0$ is small enough. As far as we know, there are no theoretical results on the stability of waves for the case when $k$ is not small and $d \neq 1$ so far.

For $d \neq 1$ and $f(v)=v^{p}$ with $p>1$, the existence of traveling front solutions of the autocatalytic system (1.1) has been investigated in $[1,6,9,10,15,19]$. For $d>0$ and $f(v)=v^{2}$, it was shown in [6] that there exists a critical speed $c_{*}(d)$ such that (1.1) has traveling waves $\left(U_{c}(x-c t), V_{c}(x-c t)\right)$ for any $c \geq c_{*}(d)$, and the waves tend to $(1,0)$ algebraically as $z \rightarrow+\infty$ for $c>c_{*}(d)$. For $0<d \leq 1$ and $f(v)=v^{p}$ with $p>1$, by applying different phase plane analysis, it was proved in $[1,10,15]$ that there exists a critical speed which is also the minimal speed $c_{*}(d)>0$ of the wave fronts, and the waves with the critical speed $c=c_{*}(d)$ decay exponentially at both ends ( see [15] ) and the waves with noncritical speeds $c>c_{*}(d)$ decay algebraically at $z=+\infty$ (see [1] and [15] ). Besides, by applying three dimensional phase plane analysis, it was also proved in [15] that the minimal speed $c_{*}(d)$ is strictly monotone decreasing in $d$ for $0 \leq d \leq 1$. For $d>1$ and $f(v)=v^{p}$ with $p>1$, the existence of waves with exponential or algebraic spatial decay was also claimed but without detailed proof in [15]. For the more general $f(v)$ including 
the case $f(v)=v^{p}$ with $p \geq 1$ or the case $f(v)=k_{1} v^{m}+k_{2} v^{n}$ with $n>m \geq 1$ and $k_{1}, k_{2}>0$, the existence and estimates of the critical speeds have been investigated in $[1,9,10,19]$, where the existence of the minimal speed for $0<d \leq 1$ and the estimates of the critical speeds of the waves for any $d>0$ were obtained. Some detailed estimates on the decaying estimates of the waves with noncritical speeds were also obtained in [1] for $0<d<1$.

For the system (1.3) with more general $f$ satisfying (1.7) or (1.10), by combining the existence results obtained in $[1,9,10,15,19]$, and some detailed spatial decaying estimates obtained in $[1,15]$ for $0<d \leq 1$, we can summarize some known existence results as follows.

Theorem 1. Assume either (1.7) or (1.10) holds.

(i) For any $d>0$ there exist some critical speeds $\bar{c}(d)$ and $\underline{c}(d)$ with $\bar{c}(d) \geq \underline{c}(d)>0$, such that there exists a traveling front solution $\left(U_{c}(z, d), V_{c}(z, d)\right)$ satisfying (1.3) for any $c \geq \bar{c}(d)$, and there is no traveling front solution for any $c<\underline{c}(d)$.

(ii) Let $0<d \leq 1$. Then $\bar{c}(d)=\underline{c}(d)=c_{\min }(d)$, and the minimal speed $c_{\min }(d)$ is monotone decreasing in $d$. Furthermore, if (1.10) holds, then the wave fronts with a noncritical speed $c>c_{\min }(d)$ decay algebraically as $z \rightarrow+\infty$ and the wave fronts with the minimal speed decay exponentially as $z \rightarrow+\infty$; while, if (1.7) holds, the wave fronts with a noncritical speed decay with a lower exponential rate $\mathrm{e}^{\sigma_{+}^{+}(c) z}$ as $z \rightarrow+\infty$ and the waves with the minimal speed decay with a higher exponential rate $\mathrm{e}^{\sigma_{+}^{-}(c) z}$ as $z \rightarrow+\infty$, where $\sigma_{+}^{ \pm}(c)=\left(-c \pm \sqrt{c^{2}-4 f^{\prime}(0)}\right) / 2$.

It is worth mentioning that the two or three dimensional phase plane analysis and the powerful comparison methods used in $[1,9,15,19]$ for the case $0<d \leq 1$ can't be applied directly to the case $d>1$. As far as we know, when $d>1$ and $f(v)$ satisfies (1.7) or (1.10), the problem about the existence of minimal speed is still open, and it is not clear if the waves with some critical speeds or noncritical speeds decay exponentially or algebraically at $z+\infty$ when (1.10) holds ( or decay with a higher or a lower exponential rate when (1.7) holds), or if the critical speed depends on $d$ continuously. In fact the precise decaying rate of the waves and the estimates of the critical speed also play an important role in the investigation of the stability of waves, especially in the spectral stability analysis of the wave.

In this paper, for system (1.3) with more general $f$ satisfying (1.7) or (1.10), by virtue of the exponential spatial decay of the waves with critical speed and the spectral stability of the waves for the system (1.3) with $d=1$, by applying special perturbation method and detailed decomposition estimates in some suitable weighted spaces, we shall first prove that when $d$ is near 1 system (1.3) has a family of wave fronts with some critical speed $c_{*}(d)$ near $c_{*}^{0}$, which decay exponentially at both ends and the critical speed $c_{*}(d)$ tends to $c_{*}^{0}$ as $d \rightarrow 1$. Further, by applying phase plane analysis and different comparison argument from those in $[1,9,15]$ as well as approximate center manifold theorem, we shall further prove the existence of traveling fronts with algebraic spatial decay for $p>1$ and $d>1$, and obtain the upper bound of $c_{*}(d)$ for $d>1$.

In the remaining of this paper, without loss of generality, we always assume $f(1)=1$.

Our main existence results in this paper can be stated as follows:

Theorem 1.1. (Existence and spatial decay of wave fronts with critical speeds) Assume either (1.10) holds or (1.7) and $c_{*}^{0}>2 \sqrt{f^{\prime}(0)}$ hold, and denote $c_{*}^{0}$ to be the minimal speed of the waves of (1.5)-(1.6). Then for each fixed $d \in[1-\delta, 1+\delta]$ with $\delta>0$ small enough, there exist a wave speed $c_{*}(d)$ and a unique (up to shift) traveling front solution $\left(U_{c_{*}(d)}(z, d), V_{c_{*}(d)}(z, d)\right)$ satisfying (1.3) and

$$
\left\{\begin{array}{l}
c_{*}(d) \rightarrow c_{*}^{0}, \\
\left\|\left(1+\mathrm{e}^{\alpha z}\right)\left(U_{c_{*}(d)}(z, d)-U_{c_{*}^{0}}(z)\right)\right\|_{L_{\infty}(\mathbb{R})}+\left\|\left(1+\mathrm{e}^{\alpha z}\right)\left(V_{c_{*}(d)}(z, d)-V_{c_{*}^{0}}(z)\right)\right\|_{L_{\infty}(\mathbb{R})} \rightarrow 0,
\end{array} \quad \text { as } d \rightarrow 1 ;\right.
$$

where $\alpha \in\left(0, c_{*}^{0} / 2\right)$ if $(1.10)$ holds, and $\alpha \in\left(\left(c_{*}^{0}-\sqrt{\left(c_{*}^{0}\right)^{2}-4 f^{\prime}(0)}\right) / 2, c_{*}^{0} / 2\right)$ if (1.7) holds. 
Furthermore, it holds that

$$
\left\{\begin{array}{l}
U_{c_{*}(d)}(z, d), V_{c_{*}(d)}(z, d)-1 \sim \mathrm{e}^{\sigma_{-}^{+}(d) z} \quad \text { as } z \rightarrow-\infty ; \\
V_{c_{*}(d)}(z, d), U_{c_{*}(d)}(z, d)-1 \sim \mathrm{e}^{-c_{*}(d) z} \quad \text { as } z \rightarrow+\infty, \text { for } 0<d \leq 1, \text { if }(1.10) \text { holds }, \\
V_{c_{*}(d)}(z, d) \sim \mathrm{e}^{-c_{*}(d) z}, U_{c_{*}(d)}(z, d)-1 \sim \mathrm{e}^{-\frac{c_{*}(d)}{d} z} \text { as } z \rightarrow+\infty, \text { for } d>1 \text {, if }(1.10) \text { holds; } \\
V_{c_{*}(d)}(z, d), U_{c_{*}(d)}(z, d)-1 \sim \mathrm{e}^{\sigma_{+}^{-}(d) z} \text { as } z \rightarrow+\infty, \text { if }(1.7) \text { holds; } \\
\text { with } \sigma_{-}^{+}(d)=\frac{-c_{*}(d)+\sqrt{c_{*}^{2}(d)+4}}{2} \text { and } \sigma_{+}^{-}(d)=\frac{-c_{*}(d)-\sqrt{c_{*}^{2}(d)-4 f^{\prime}(0)}}{2}
\end{array}\right.
$$

Theorem 1.2. (Existence and spatial decay of wave fronts with noncritical speeds) Assume either (1.10) or (1.7) holds, let $\left(U_{c_{*}(d)}(z, d), V_{c_{*}(d)}(z, d)\right)$ be a wave front with the speed $c_{*}(d)$ to system (1.3) satisfying (1.12), then

$$
\text { (i) } c_{*}(d) \geq c_{*}^{0}, \text { if } 0 \leq d<1 ; \quad c_{*}(d) \leq c_{*}^{0} \text {, if } d>1 .
$$

(ii) For $c>c_{*}(d)$ if $0<d \leq 1$ or for $c \geq c_{*}^{0}$ if $d>1$ and (1.10) holds; or for $c>c_{*}^{0}$ if $d>1$ and (1.7) holds, then there exists a unique traveling front solution $\left(U_{c}(z, d), V_{c}(z, d)\right)$ to system (1.3), which satisfies the following decaying estimates

$$
\left\{\begin{array}{l}
U_{c}(z, d), \quad 1-V_{c}(z, d) \sim \mathrm{e}^{\sigma_{-}^{+}(c) z} \text { as } z \rightarrow-\infty ; \\
V_{c}(z, d), \quad 1-U_{c}(z, d) \sim k z^{-\frac{1}{p-1}} \quad \text { as } z \rightarrow+\infty, \text { if }(1.10) \text { holds }, \\
V_{c}(z, d), \quad 1-U_{c}(z, d) \sim \mathrm{e}^{\sigma_{+}^{+}(c) z} \text { as } z \rightarrow+\infty, \text { if }(1.7) \text { holds } \\
\text { with } k=\left(\frac{p-1}{c}\right)^{\frac{1}{1-p}}, \sigma_{-}^{+}(c)=\frac{-c+\sqrt{c^{2}+4}}{2} \text { and } \sigma_{+}^{+}(c)=\frac{-c+\sqrt{c^{2}-4 f^{\prime}(0)}}{2}
\end{array}\right.
$$

Remark 1.3. Under the assumption of (1.10), Theorem 1.1 and Theorem 1.2 guarantee the existence of wave fronts with some critical speed $c_{*}(d)\left(<c_{*}^{0}\right)$ and with exponential decay for $d>1$ and $d$ near 1 , and the existence of wave fronts with algebraic decay for any $d>1$ and $c \geq c_{*}^{0}$. However it is not clear if there exist wave fronts with speed $c \in\left(c_{*}(d), c_{*}^{0}\right)$ when $d>1$ even if $d$ is near 1 , which is still an open problem. It is worth mentioning that in [15] under the assumption of (1.10), for any $d>1$ the existence of minimal speed and similar results about the existence of waves with exponential decay and algebraic decay are also claimed in [15] but without detailed proof. In this paper by applying different method of proof and different phase plane analysis from those in $[1,9,15]$, we shall first investigate the existence and the precise decaying rates of waves for $d \neq 1$ and for more general $f$ covering (1.10) and (1.7), which will be useful in our later investigation of the stability of the wave.

For the case (1.10) when $d$ is near 1, by applying spectral method and detailed semigroup estimates in some exponentially weighted or unweighted spaces, the linear and nonlinear asymptotic stability of the wave fronts with noncritical speeds $c>\max \left\{c_{*}(d), c_{*}^{0}\right\}$ and with algebraic spatial decay was recently obtained in [21], where more general Evans function theories were also established and applied to prove the spectral stability of the waves with algebraic spatial decay for $d$ near 1 .

In this paper for the case (1.7) and the case (1.10) we shall further investigate the stability of waves with critical speeds and with exponential spatial decay for system (1.1) when $d$ is near 1. By virtue of the exponential decaying estimates of the waves with the critical speeds $c_{*}(d)$ and the continuity of $c_{*}(d)$ for $d$ near 1 , by applying spectral analysis and standard Evans function method, we shall prove the spectral stability of the waves with critical speeds in some exponentially weighted spaces. Further, by combining the main idea of proof in [12] with some similar estimates in [21], we shall prove the nonlinear asymptotic stability of the waves with the critical or noncritical speeds in some suitable weighted or unweighted spaces. 
Let $\left(U_{c}(x-c t), V_{c}(x-c t)\right)$ be a traveling front solution of $(1.3)$, in moving coordinate $z=x-c t$ the system (1.1) with initial data $\left(u_{0}, v_{0}\right)$ becomes

$$
\begin{cases}u_{t}(z, t)=d u_{z z}(z, t)+c u_{z}(z, t)-u f(v), & z \in \mathbb{R}, t>0 \\ v_{t}(z, t)=v_{z z}(z, t)+c v_{z}(z, t)+u f(v), & z \in \mathbb{R}, t>0 \\ \left.u\right|_{t=0}=u_{0}(z),\left.\quad v\right|_{t=0}=v_{0}(z), & z \in \mathbb{R}\end{cases}
$$

For the fixed pair $\left(\alpha^{-}, \alpha^{+}\right) \triangleq \alpha$ with $\alpha_{-} \geq 0$ and $\alpha_{+}>0$, define weight function $w_{\alpha}(z) \in C^{2}(\mathbb{R})$ satisfying

$$
w_{\alpha}(z)= \begin{cases}\mathrm{e}^{\alpha^{-} z}, & z \leq 0 \\ \in\left[1, \mathrm{e}^{\alpha^{+}}\right], & z \in(0,1) \\ \mathrm{e}^{\alpha^{+} z}, & z \geq 1\end{cases}
$$

and define the weighted space $C_{\alpha}(\mathbb{R})=\left\{v(z) \mid v(z) w_{\alpha}(z) \in C_{\text {unif }}(R)\right\}$ with norm $\|v\|_{C_{\alpha}}=$ $\left\|v(z) w_{\alpha}(z)\right\|_{L_{\infty}}, C_{\alpha}^{2}(\mathbb{R})$ can be defined similarly. For the case $\alpha^{-}=0$ and $\alpha^{+}>0$, the weight function $w_{\alpha}(z)$ defined in (1.16) can be replaced by $w_{\alpha}(z)=1+\mathrm{e}^{\alpha^{+} z}$, and denote the corresponding weighted space $C_{\alpha}(\mathbb{R})$ by $\mathcal{B}_{\alpha^{+}}(\mathbb{R})$ and $\mathcal{B}_{\alpha^{+}}^{2}(\mathbb{R})$ can be defined similarly; and define $X_{\alpha}=C_{\alpha}(\mathbb{R}) \times C_{\alpha}(\mathbb{R})$ and $\mathcal{Z}_{\alpha^{+}}=\mathcal{B}_{\alpha^{+}}(\mathbb{R}) \times \mathcal{B}_{\alpha^{+}}(\mathbb{R})$.

Now we state our nonlinear stability results as follows:

Theorem 1.4. (Nonlinear stability of wave fronts with the critical speeds) Assume either (1.10) holds or (1.7) and $c_{*}^{0}>2 \sqrt{f^{\prime}(0)}$ hold; and let $\left(U_{c_{*}}(z, d), V_{c_{*}}(z, d)\right)\left(z=x-c_{*}(d) t\right)$ be the traveling front with the critical speed $c_{*}(d)$ obtained in Theorem 1.1 for $d$ near 1; and $c_{*}^{0}$ be the minimal speed of the wave fronts of (1.5)-(1.6).

(i) For each fixed $\alpha^{+} \in\left(\alpha_{-}^{0}, \alpha_{+}^{0}\right)$ and small $\alpha^{-}>0$, with $\alpha_{-}^{0}=\left(c_{*}^{0}-\sqrt{\left(c_{*}^{0}\right)^{2}-4 f^{\prime}(0)}\right) / 2 \geq 0\left(\alpha_{-}^{0}=0\right.$ if (1.7) holds) and $\alpha_{+}^{0}=c_{*}^{0} / 2$; there exist positive constants $\delta_{0}, \delta_{\alpha}^{0}, \sigma_{\alpha}$ and $K$ and some constant $\gamma_{*} \in \mathbb{R}$ such that for any $d \in\left[1-\delta_{0}, 1+\delta_{0}\right]$ if

$$
\left\|\left(u_{0}(z)-U_{c_{*}}(z), v_{0}(z)-V_{c_{*}}(z)\right)\right\|_{\mathcal{Z}_{\alpha+}} \leq \delta_{\alpha}^{0},
$$

then the solution to initial value problem (1.15) satisfies

$$
\mathrm{e}^{\sigma_{\alpha} t / 2}\left\|u(z, t)-U_{c_{*}}\left(z+\gamma_{*}\right)\right\|_{C_{u n i f}(\mathbb{R})}+\left\|v(z, t)-V_{c_{*}}\left(z+\gamma_{*}\right)\right\|_{C_{u n i f}(\mathbb{R})} \leq K \delta_{\alpha}^{0}, \quad \forall t \geq 0,
$$

and

$$
\left\|\left(u(z, t)-U_{c_{*}}\left(z+\gamma_{*}\right), v(z, t)-V_{c_{*}}\left(z+\gamma_{*}\right)\right)\right\|_{X_{\alpha}} \leq K \delta_{\alpha}^{0} \mathrm{e}^{-\sigma_{\alpha} t}, \quad \forall t \geq 0 .
$$

(ii) Under the assumption of (i), if $\delta_{\alpha}^{0}>0$ is small enough and

$$
\left\|u_{0}-U_{c_{*}}\right\|_{L_{1}(\mathbb{R})}+\left\|v_{0}-V_{c_{*}}\right\|_{L_{1}(\mathbb{R})} \leq \delta_{\alpha}^{0},
$$

then we further have

$$
\mathrm{e}^{\sigma_{\alpha} t / 2}\left\|u(z, t)-U_{c_{*}}\left(z+\gamma_{*}\right)\right\|_{C_{\text {unif }}(\mathbb{R})}+\sqrt{1+t}\left\|v(z, t)-V_{c_{*}}\left(z+\gamma_{*}\right)\right\|_{C_{\text {unif }}(\mathbb{R})} \leq K_{1} \delta_{\alpha}^{0}, \quad \forall t \geq 0,
$$

and

$$
\mathrm{e}^{\sigma_{\alpha} t / 2}\left\|u(z, t)-U_{c_{*}}\left(z+\gamma_{*}\right)\right\|_{L_{1}(\mathbb{R})}+\left\|v(z, t)-V_{c_{*}}\left(z+\gamma_{*}\right)\right\|_{L_{1}(\mathbb{R})} \leq K_{1} \delta_{\alpha}^{0}, \quad \forall t \geq 0,
$$

with constant $K_{1}$ independent of $t$ and $\delta_{\alpha}^{0}$.

Theorem 1.5. (Nonlinear stability of wave fronts with noncritical speeds) Assume (1.7) holds, let $c_{*}^{0}$ be the minimal speed of waves of (1.5) satisfying (1.6), then for each fixed $c>c_{*}^{0}$ there exists a small positive constant $\delta_{0}$, such that for any $d \in\left[1-\delta_{0}, 1+\delta_{0}\right]$ the solution $(u(z, t), v(z, t))$ of system (1.15) with a small initial perturbation of the wave front $\left(U_{c}(z, d), V_{c}(z, d)\right)$ satisfies all the estimates stated in Theorem 1.4, with $c_{*}(d)$ being replaced by $c$ and $\gamma_{*}=0$. 
Remark 1.6. The estimates obtained in Theorem 1.4 guarantee that under the assumption of (1.10) or (1.7) with $c_{*}^{0}>2 \sqrt{f^{\prime}(0)}$, if $d$ is near 1 and if the initial perturbation of a wave front with the critical speed $c_{*}(d)$ is small in $L_{1}(\mathbb{R}) \cap C_{\text {unif }}(\mathbb{R}) \cap C_{\alpha}(\mathbb{R})$ for some suitable positive $\alpha^{ \pm}$, then the perturbation of the wave front always stays small in $L_{1}$ norm and decays to zero algebraically in $C_{\text {unif }}(\mathbb{R})$ norm and decays exponentially in $C_{\alpha}(\mathbb{R})$ norm. Under the assumption of $(1.10)$, similar nonlinear stability results but without shift were also obtained in [21] for the waves with noncritical speeds $c>c_{*}^{0}$ when $d$ is near 1, where the algebraic spatial decaying of the waves with noncritical speeds and selection of the initial perturbation in the spaces $C_{\text {unif }}(\mathbb{R}) \cap C_{\alpha}(\mathbb{R})$ with small $\alpha^{-}=\alpha^{+}>0$, require that the initial value tends to $(1,0)$ at one end algebraically with the same algebraic decaying rate of the wave. The nonlinear stability of the waves with critical speeds stated in Theorem 1.4 requires that the initial value $\left(u_{0}, v_{0}\right)$ tend to $(1,0)$ exponentially at one end. For the case (1.10) the nonlinear stability (with shift) of the waves with exponential spatial decay obtained in Theorem 1.4 and the nonlinear stability results (without shift) obtained in [21] for the waves with algebraic spatial decay also imply that the decaying rate of the initial value at $z=\infty$ may determine the asymptotic speed of the level set of solution or the asymptotic behavior of the solution when $d$ is near 1 , which is true for the special case when $d=1$, see [29] [30] and [32].

For the system with a mixed order reaction $f(v)=v+k v^{p}$ for $k \geq 0$ and $p \geq 2$, Theorem 1.4 and Theorem 1.5 guarantee that for any $k \geq 0, p \geq 2$ and $d$ near 1 , all the waves with speed $c>2 \sqrt{f^{\prime}(0)}=2$ are nonlinearly asymptotically stable in both $C_{\text {unif }}(\mathbb{R})$ and $C_{\alpha}(\mathbb{R})$, if the initial perturbation of the wave is small in $L_{1}(\mathbb{R}) \cap C_{\text {unif }}(\mathbb{R}) \cap C_{\alpha}(\mathbb{R})$ for small $\alpha^{-}>0$ and some suitable $\alpha^{+}>0$, and the perturbation decays to zero in time algebraically in $C_{\text {unif }}(\mathbb{R})$ norm and decays exponentially in $C_{\alpha}(\mathbb{R})$ norm, which improves the nonlinear asymptotic stability results obtained in [11], except the special case when $c_{*}(d)=2$. For the case $f(v)=v+k v^{2}$ with $k \geq 0$ small enough and $d$ near 1, in [11] by energy method the waves with speeds $c \geq 2$ are proved to be nonlinearly asymptotic stable (without decaying estimates) in some weighted spaces $\mathcal{B}_{\alpha^{+}}(\mathbb{R})$.

The rest of this paper is organized as follows. The proof of Theorems 1.1 and 1.2 will be given in Section 2. The linear exponential stability of the waves in some exponentially weighted spaces will be proved in Section 3. In Section 4 we shall prove the nonlinear stability results stated in Theorems 1.4 and 1.5 .

\section{The existence and spatial decay of traveling fronts for $d \neq 1$}

\subsection{The existence of traveling waves with exponential decay}

Let $(U(x-c t), V(x-c t))$ be a traveling wave of $(1.1)$ connecting $(0,1)$ and $(1,0)$, then $(U(z), V(z))$ satisfies

$$
\left\{\begin{array}{l}
d U^{\prime \prime}+c U^{\prime}-U f(V)=0, \\
V^{\prime \prime}+c V^{\prime}+U f(V)=0,
\end{array}\right.
$$

and

$$
\left\{\begin{array}{l}
U(-\infty)=0, U(\infty)=1 \\
V(-\infty)=1, V(\infty)=0 .
\end{array}\right.
$$

Let $W(z)=U(z)+V(z)-1$, then $(W(z), V(z))$ satisfies

$$
\left\{\begin{array}{l}
d W^{\prime \prime}+c W^{\prime}+(1-d) V^{\prime \prime}=0, \\
V^{\prime \prime}+c V^{\prime}+(1-V+W) f(V)=0,
\end{array} \quad z \in \mathbb{R} ;\right.
$$

and

$$
\left\{\begin{array}{l}
W(-\infty)=0, W(\infty)=0, \\
V(-\infty)=1, V(\infty)=0 .
\end{array}\right.
$$


Obviously, system (2.3)-(2.4) can be rewritten as

$$
\left\{\begin{array}{l}
W^{\prime}=-\frac{c}{d} W+\delta_{d} V^{\prime} \\
V^{\prime \prime}=-c V^{\prime}-(1-V+W) f(V), \\
W(-\infty)=0, \quad W(\infty)=0 \\
V(-\infty)=1, \quad V(\infty)=0
\end{array}\right.
$$

where $\delta_{d}=(d-1) / d$.

(2.5) further implies that

$$
W(z)=\delta_{d} \int_{-\infty}^{z} \mathrm{e}^{\frac{c}{d}(t-z)} V^{\prime}(t) d t=\delta_{d} V(z)-\frac{c}{d} \delta_{d} \int_{-\infty}^{z} \mathrm{e}^{\frac{c}{d}(t-z)} V(t) d t .
$$

It can be checked that if $|V(z)| \leq C_{0}$ for $z \in \mathbb{R}$ and $\int_{R}\left|V^{\prime}(t)\right| d t$ is convergent, then (2.6) further implies that

$$
W(z)=\delta_{d} a(z, V(z)),|a(z, V(z))| \leq 2 C_{0}, \quad \forall z \in \mathbb{R} ; \text { and } a( \pm \infty, V)=0 ;
$$

where

$$
a(z, V(z))=V(z)-\frac{c}{d} \int_{-\infty}^{z} \mathrm{e}^{\frac{c}{d}(t-z)} V(t) d t
$$

thus (2.5) is equivalent to the following scalar integro-differential equation:

$$
\left\{\begin{array}{l}
V^{\prime \prime}=-c V^{\prime}-\left(1-V+\delta_{d} a(z, V)\right) f(V), \quad z \in \mathbb{R} \\
V(-\infty)=1, \quad V(+\infty)=0
\end{array}\right.
$$

For each fixed $d>0$, let $V_{c}(z, d)$ be the solution of (2.8) satisfying $0 \leq V_{c}(z, d) \leq 1$ and $V_{c}^{\prime}(z, d) \leq 0$ for $z \in \mathbb{R}$. Then, by standard asymptotic analysis and applying center manifold theorem, it can be proved that $V_{c}(z, d)$ satisfies

$$
\begin{gathered}
1-V_{c}(z, d), V_{c}^{\prime}(z, d) \rightarrow 0 \text { exponentially as } z \rightarrow-\infty \\
\mathrm{e}^{c z} V_{c}(z, d) \rightarrow C_{0}>0, \mathrm{e}^{c z} V_{c}^{\prime}(z, d) \rightarrow-c C_{0} \text { as } z \rightarrow+\infty
\end{gathered}
$$

or

$$
V_{c}(z, d), V_{c}^{\prime}(z, d) \rightarrow 0 \text { algebraically as } z \rightarrow+\infty .
$$

If $V_{c}(z, d) \rightarrow 0$ exponentially as $z \rightarrow+\infty$, then by (2.6), (2.7), (2.9) and (2.10), we have

$$
\begin{aligned}
\left|W\left(z, V_{c}\right)\right| & \leq \delta_{d} \int_{-\infty}^{z} \mathrm{e}^{\frac{c}{d}(t-z)}\left|V_{c}^{\prime}(t, d)\right| d t \leq \delta_{d}\left(\mathrm{e}^{-\frac{c}{d} z} \int_{-\infty}^{1} \mathrm{e}^{\frac{c}{d} t}\left|V_{c}^{\prime}(t, d)\right| d t+\mathrm{e}^{-\frac{c}{d} z} \int_{1}^{z} \mathrm{e}^{\frac{c}{d} t}\left|V_{c}^{\prime}(t, d)\right| d t\right) \\
& \leq C \delta_{d}\left(\mathrm{e}^{-\frac{c}{d} z}+\mathrm{e}^{-c z}\right), \quad \text { if } z \geq 1 \text { and } d \neq 1 .
\end{aligned}
$$

In the remaining part of this section, under the assumption (1.7) or (1.10), we shall prove the existence of solution of (2.8) which decays exponentially at both ends. Let $V_{*}(z)$ be the traveling wave of $(1.5)$ with the minimal speed $c=c_{*}^{0}$, i.e. $V_{*}(z)$ satisfies

$$
\left\{\begin{array}{l}
V_{*}^{\prime \prime}+c_{*}^{0} V_{*}^{\prime}+\left(1-V_{*}\right) f\left(V_{*}\right)=0, \quad z \in \mathbb{R} \\
V_{*}(-\infty)=1, \quad V_{*}(+\infty)=0
\end{array}\right.
$$

Let $\hat{v}(z)=V_{c}(z, d)-V_{*}(z)$ and $\hat{c}=c-c_{*}^{0}$, then (2.8) becomes

$$
\left\{\begin{array}{l}
\hat{v}^{\prime \prime}+c \hat{v}^{\prime}+b(z) \hat{v}+\hat{c} V_{*}^{\prime}+g(\hat{v})+\delta_{d} a\left(z, V_{*}+\hat{v}\right) f\left(V_{*}+\hat{v}\right)=0, \quad z \in \mathbb{R} \\
\hat{v}( \pm \infty)=0
\end{array}\right.
$$

with $b(z)=\left.\frac{\partial f_{1}(v)}{\partial v}\right|_{v=V_{*}}, f_{1}(v)=(1-v) f(v)$ and $g(\hat{v})=f_{1}\left(V_{*}+\hat{v}\right)-f_{1}\left(V_{*}\right)-b(z) \hat{v}$. 
Note that

$$
\left\{\begin{array}{ll}
|g(\hat{v})| \leq C|\hat{v}|^{2}, & \text { for case }(1.10) \text { with } p \geq 2 \text { or for case }(1.7), \\
|g(\hat{v})| \leq C|\hat{v}|^{p}, & \text { for case }(1.10) \text { with } 1<p<2
\end{array} \text { if }|\hat{v}| \leq 1\right.
$$

Define operator

$$
L=\frac{\partial^{2}}{\partial z^{2}}+c_{*}^{0} \frac{\partial}{\partial z}+b(z),
$$

then (2.14) can be written as

$$
L \hat{v}=F\left(\hat{v}, \hat{c}, \delta_{d}\right) \triangleq-\hat{c} V_{*}^{\prime}-\hat{c} \hat{v}^{\prime}-g(\hat{v})-\delta_{d} a\left(z, V_{*}+\hat{v}\right) f\left(V_{*}+\hat{v}\right) .
$$

For each fixed $\alpha>0$, define weighted space $\mathcal{B}_{\alpha}(R)=\left\{v(z) \mid v(z)\left(1+\mathrm{e}^{\alpha z}\right) \in C_{\text {unif }}(R)\right\}$ with norm $\|v\|_{\mathcal{B}_{\alpha}}=\left\|v(z)\left(1+\mathrm{e}^{\alpha z}\right)\right\|_{L_{\infty}}$, and $\mathcal{B}_{\alpha}^{2}(R)$ can be defined similarly.

For each fixed $\alpha>0$ define operator $L_{\alpha}: \mathcal{B}_{\alpha}^{2}(R) \rightarrow \mathcal{B}_{\alpha}(R)$ by

$$
L_{\alpha} v=L v \text {, for } v \in \mathcal{B}_{\alpha}^{2}(R) \text {. }
$$

Lemma 2.1. Assume either (1.10) holds or (1.7) and $c_{*}^{0}>2 \sqrt{f^{\prime}(0)}$ hold, then for each fixed $\alpha \in$ $\left(\alpha_{-}^{0}, \alpha_{+}^{0}\right)$, with $\alpha_{-}^{0}=\left(c_{*}^{0}-\sqrt{\left(c_{*}^{0}\right)^{2}-4 f^{\prime}(0)}\right) / 2$ and $\alpha_{+}^{0}=c_{*}^{0} / 2$, there exists $\delta_{\alpha}>0$ such that

$$
\text { (i) } \sup \left\{\operatorname{Re}\left\{\sigma\left(L_{\alpha}\right) \backslash\{0\}\right\}\right\} \leq-\delta_{\alpha} ;
$$

(ii) zero is a simple eigenvalue of $L_{\alpha}$ with eigenspace $\operatorname{Ker}\left(L_{\alpha}\right)$ spanned by $V_{*}^{\prime}(z)$.

Proof. Under the assumption of Lemma 2.1, by applying the standard spectral analysis on $L_{\alpha}$, it can be proved that for each fixed $\alpha \in\left(\alpha_{-}^{0}, \alpha_{+}^{0}\right)$ there exists small $\delta_{\alpha}>0$ such that

$$
\operatorname{Re} \sigma_{\text {ess }}\left(L_{\alpha}\right)<-\delta_{\alpha}<0 .
$$

Further, by virtue of the exponential decay of $V_{*}^{\prime}(z)$ at both ends for the case (1.10), or by the higher exponential decaying rate of wave $V_{*}^{\prime}(z)$ for the case (1.7) if $c_{*}^{0}>2 \sqrt{f^{\prime}(0)}$, we can apply the standard spectral arguments including the application of Sturm-Liuville theorem to prove that all the eigenvalues located in $\left\{\lambda \in \mathbb{C} \mid \operatorname{Re} \lambda \geq-\delta_{\alpha}\right\}$ are real, which are also the isolated eigenvalues of some self-adjoint second order linear differential operator, thus zero is the first and a simple eigenvalue of $L_{\alpha}$. The detailed proof is essentially the same as that in [30], in which the results of Lemma 2.1 are proved for the case $f(v)=v^{p}$ with $p>1$. Here we omit the details of the proof.

Lemma 2.1 implies that

$$
\mathcal{B}_{\alpha}(\mathbb{R})=\operatorname{Ker}\left(L_{\alpha}\right) \oplus \operatorname{Range}\left(L_{\alpha}\right) .
$$

Define the projection $P: \mathcal{B}_{\alpha}(\mathbb{R}) \rightarrow \operatorname{Ker}\left(L_{\alpha}\right)$ by

$$
P v=\frac{<v, \phi_{1}>}{<V_{*}^{\prime}, \phi_{1}>} V_{*}^{\prime},
$$

where $\phi_{1}(z)=\mathrm{e}^{c_{*}^{0} z} V_{*}^{\prime}(z)$ and $\langle u, v\rangle=\int_{R} u(z) v(z) d z$.

Notice that $\left\langle v, \phi_{1}\right\rangle$ is finite for any $v \in \mathcal{B}_{\alpha}(\mathbb{R})$ and $\phi_{1}(z)$ satisfies

$$
L^{*} \phi_{1} \triangleq \phi_{1}^{\prime \prime}(z)-c_{*}^{0} \phi_{1}^{\prime}(z)+b(z) \phi_{1}(z) \equiv 0,
$$

then it is easy to see that $\left.<v, \phi_{1}\right\rangle=0, \forall v \in \operatorname{Range}\left(L_{\alpha}\right)$. Furthermore, using (2.18) and the fact that $<V_{*}^{\prime}, \phi_{1}>\neq 0$, we have Range $\left(L_{\alpha}\right)=\left\{\phi_{1}\right\}^{\perp} \cap \mathcal{B}_{\alpha}(\mathbb{R})$. Thus

$$
(I-P) v=v-\frac{<v, \phi_{1}>}{<V_{*}^{\prime}, \phi_{1}>} V_{*}^{\prime} \in \operatorname{Range}\left(L_{\alpha}\right), \quad \forall v \in \mathcal{B}_{\alpha}(\mathbb{R})
$$


Proof of Theorem 1.1. Under the assumption of Theorem 1.1, for a fixed $\alpha \in\left(\alpha_{-}^{0}, \alpha_{+}^{0}\right)$, let $\hat{v}(z)$ be the solution of nonlinear equation $(2.16)$ in $\mathcal{B}_{\alpha}^{2}(R)$ with small $\|\hat{v}\|_{\mathcal{B}_{\alpha}}$, i.e. $\hat{v}$ satisfies

$$
L \hat{v}=-\hat{c} V_{*}^{\prime}-\hat{c} \hat{v}^{\prime}-g(\hat{v})-\delta_{d} a\left(z, V_{*}+\hat{v}\right) f\left(V_{*}+\hat{v}\right) \triangleq F\left(\hat{v}, \hat{c}, \delta_{d}\right), \text { and } \hat{v} \in \mathcal{B}_{\alpha}^{2}(R),
$$

which implies that $<F\left(\hat{v}, \hat{c}, \delta_{d}\right), \phi_{1}>=0$, thus $\hat{c}$ can be uniquely determined by

$$
\hat{c}=\frac{<-g(\hat{v})-\delta_{d} a\left(z, V_{*}+\hat{v}\right) f\left(V_{*}+\hat{v}\right), \phi_{1}>}{<V_{*}^{\prime}+\hat{v}^{\prime}, \phi_{1}>} .
$$

Define operator

$$
L^{\perp}: D\left(L^{\perp}\right)=\text { Range }(L) \cap \mathcal{B}_{\alpha}^{2}(R) \rightarrow X_{2}=\operatorname{Range}(L) \cap C_{\alpha}(R)
$$

by

$$
L^{\perp} \hat{v}=L \hat{v}, \quad \text { for } v \in D\left(L^{\perp}\right),
$$

with $\|v\|_{D\left(L^{\perp}\right)}=\|v\|_{\mathcal{B}_{\alpha}^{2}}$ and $\|v\|_{X_{2}}=\|v\|_{\mathcal{B}_{\alpha}}$.

Lemma 2.1 and (2.18) imply that $L^{\perp}$ is invertible with the bounded inverse $\left(L^{\perp}\right)^{-1}$, consider the following nonlinear equation:

$$
\hat{v}=\left(L^{\perp}\right)^{-1} F^{\perp}\left(\hat{v}, \delta_{d}\right) \triangleq G\left(\hat{v}, \delta_{d}\right)
$$

with

$$
F^{\perp}\left(\hat{v}, \delta_{d}\right)=-g(\hat{v})-\delta_{d} a\left(z, V_{*}+\hat{v}\right) f\left(V_{*}+\hat{v}\right)+\frac{<g(\hat{v})+\delta_{d} a\left(z, V_{*}+\hat{v}\right) f\left(V_{*}+\hat{v}\right), \phi_{1}>}{<V_{*}^{\prime}+\hat{v}^{\prime}, \phi_{1}>}\left(V_{*}^{\prime}+\hat{v}^{\prime}\right) \in X_{2} .
$$

It is easy to check that if $\hat{v} \in D\left(L^{\perp}\right)$ is a solution of (2.24) with small enough $\|\hat{v}\|_{C_{\alpha}(R)}$ and $\hat{c}$ satisfies $(2.22)$, then $(\hat{v}(z), \hat{c})$ satisfies $(2.21)$.

In the following we shall prove that if $|d-1|$ is small enough then there exists a unique solution $(\hat{v}(z), \hat{c})$ satisfying (2.24) and (2.22).

For $d=1$ i.e. $\delta_{d}=0$, obviously $G(0,0)=0$ and $(\hat{v}, \hat{c})=(0,0)$ is a solution of $(2.24)$ and $(2.22)$.

For any $\delta_{d}$ and any $\hat{v}_{1}, \hat{v}_{2} \in X_{2}$ satisfying $\left|\delta_{d}\right|,\left\|\hat{v}_{1}\right\|_{\mathcal{B}_{\alpha}},\left\|\hat{v}_{2}\right\|_{\mathcal{B}_{\alpha}} \leq \delta_{*}$ with $\delta_{*}$ small enough, by detailed computation it can be verified that

$$
\begin{gathered}
\left\|g\left(\hat{v}_{1}\right)-g\left(\hat{v}_{2}\right)\right\|_{\mathcal{B}_{\alpha}} \leq\left\{\begin{array}{l}
C\left(\left\|\hat{v}_{1}\right\|_{L_{\infty}}+\left\|\hat{v}_{2}\right\|_{L_{\infty}}\right)\left\|\hat{v}_{1}-\hat{v}_{2}\right\|_{\mathcal{B}_{\alpha}}, \quad \text { for case }(1.10) \text { with } p \geq 2 \text { or for case }(1.7), \\
\left.C_{p}\left(\left\|\hat{v}_{1}\right\|_{L_{\infty}}+\left\|\hat{v}_{2}\right\|_{L_{\infty}}\right)^{p-1}\left\|\hat{v}_{1}-\hat{v}_{2}\right\|_{\mathcal{B}_{\alpha}}\right), \text { for case }(1.10) \text { with } 1<p<2 ;
\end{array}\right. \\
\quad \text { and }\left\|a\left(z, V_{*}+\hat{v}_{1}\right)-a\left(z, V_{*}+\hat{v}_{2}\right)\right\|_{\mathcal{B}_{\alpha} \leq C\left\|\hat{v}_{1}-\hat{v}_{2}\right\|_{\mathcal{B}_{\alpha}},}
\end{gathered}
$$

with $C_{p}$ and $C$ independent of $\delta_{d}, \hat{v}_{1}$ and $\hat{v}_{2}$.

Thus we have

$$
\begin{aligned}
& \left\|G\left(\hat{v}_{1}, \delta_{d}\right)-G\left(\hat{v}_{2}, \delta_{d}\right)\right\|_{\mathcal{B}_{\alpha}^{2}} \leq C_{0}\left\|F^{\perp}\left(\hat{v}_{1}, \delta_{d}\right)-F^{\perp}\left(\hat{v}_{2}, \delta_{d}\right)\right\|_{\mathcal{B}_{\alpha}} \\
& \leq C_{0}\left\|g\left(\hat{v}_{1}\right)-g\left(\hat{v}_{2}\right)\right\|_{\mathcal{B}_{\alpha}}+\left|\delta_{d}\right|\left\|a\left(z, V_{*}+\hat{v}_{1}\right)\left(V_{*}+\hat{v}_{1}\right)^{p}-a\left(z, V_{*}+\hat{v}_{2}\right)\left(V_{*}+\hat{v}_{2}\right)^{p}\right\|_{\mathcal{B}_{\alpha}} \\
& \leq \theta\left\|\hat{v}_{1}-\hat{v}_{2}\right\|_{\mathcal{B}_{\alpha}}, \text { for some } \theta<1, \text { if } \delta_{*}>0 \text { is small enough. }
\end{aligned}
$$

We can similarly prove that $G\left(\hat{v}_{1}, \delta_{d}\right)$ is continuous in $\delta_{d}$. Then, by contraction mapping theorem, it follows that for each fixed small enough $\delta_{d}$ there exists a unique small $\hat{v}(z, d) \in D\left(L^{\perp}\right)$ and $\hat{c}(d)$ satisfying $(2.24)$ and $(2.22)$, and

$$
\|\hat{v}(z, d)\|_{D\left(L^{\perp}\right)} \rightarrow 0, \quad \hat{c}(d) \rightarrow 0, \text { as } d \rightarrow 1 .
$$

Thus, for small enough $|d-1|$ there exists a traveling wave $\left(V_{c_{*}}(z, d), W_{c_{*}}(z, d)\right) \in \mathcal{B}_{\alpha}^{2}(R) \times \mathcal{B}_{\alpha}^{2}(R)$ satisfying (2.5), where $V_{c_{*}}(z, d)=V_{*}(z)+\hat{v}(z, d), W_{c_{*}}(z, d)=\delta_{d} a\left(z, V_{c_{*}}(z, d)\right)$ and $c_{*}(d)=c_{*}^{0}(p)+\hat{c}(d)$. Let $U_{c_{*}}(z, d)=1-W_{c_{*}}(z, d)-V_{c_{*}}(z, d)$, then $\left(U_{c_{*}}(z, d), V_{c_{*}}(z, d)\right)$ is a traveling wave of $(2.1)$, and (1.11) follows from (2.25). Further, as proved in [6] $U_{c_{*}}(z, d)$ must be strictly increasing in $z$ and $V_{c_{*}}(z, d)$ strictly decreasing in $z$, then (1.12) follows from (1.8), (1.11), (2.9), (2.10) and (2.12). This completes the proof of Theorem 1.1. 


\subsection{The existence and spatial decay of traveling waves with noncritical speeds}

For each fixed $c>0$, let $(U(z), V(z))$ be a solution of $(2.1)$ satisfying $(U(-\infty), V(-\infty))=(0,1)$, with $U(z)$ being strictly increasing and $V(z)$ strictly deceasing, then $(U(z), V(z))$ also satisfies

$$
\left\{\begin{array}{l}
d U^{\prime}=-V^{\prime}-c(U+V-1) \\
V^{\prime \prime}+c V^{\prime}+U f(V)=0 \\
U(-\infty)=0, V(-\infty)=1, \\
U(z)>0, \quad V(z)<1, V^{\prime}(z)<0, \text { for } z>-\infty
\end{array}\right.
$$

Rewrite (2.26) as the following first order differential system

$$
\left\{\begin{array}{l}
U^{\prime}=-\frac{V_{2}}{d}-\frac{c}{d}\left(U+V_{1}-1\right) \\
V_{1}^{\prime}=V_{2}, \\
V_{2}^{\prime}=-c V_{2}-U f\left(V_{1}\right) \\
U(-\infty)=0, V_{1}(-\infty)=1, V_{2}(-\infty)=0, \\
U(z)>0, \quad 0<V_{1}(z)<1, V_{2}(z)<0, \text { for } z>-\infty
\end{array}\right.
$$

Linearizing the system of $(2.27)$ around $(0,1,0)$, we have

$$
Y^{\prime}=A^{-} Y, \text { with } A^{-}=\left(\begin{array}{ccc}
-\frac{c}{d} & -\frac{c}{d} & -\frac{1}{d} \\
0 & 0 & 1 \\
-1 & 0 & -c
\end{array}\right)
$$

Note that matrix $A^{-}$has two negative eigenvalues and a positive eigenvalue denoted by $\sigma_{1}(d, c)=$ $\left(-c+\sqrt{c^{2}+4 d f(1)}\right) /(2 d)$, with an eigenvector $\boldsymbol{Y}_{1}^{-}=\left(-\sigma_{2}(d, c), 1, \sigma_{1}(d, c)\right)^{T}$, where $\sigma_{2}(d, c)=$ $\sigma_{1}(d, c)\left(\sigma_{1}(d, c)+c\right)$. Then, by standard asymptotic analysis, it follows that for each fixed $c>0$ and $d>0$ there exists a unique solution $\left(U^{-}(z, d), V_{1}^{-}(z, d), V_{2}^{-}(z, d)\right)$ of $(2.27)$ satisfying

$$
\mathrm{e}^{-\sigma_{1}(d, c) z}\left(U^{-}(z, d), V_{1}^{-}(z, d)-1, V_{2}^{-}(z, d)\right)^{T} \rightarrow-\boldsymbol{Y}_{1}^{-},
$$

and any solution of $(2.27)$ can be expressed as $\left(U^{-}\left(z+z_{0}, d\right), V_{1}^{-}\left(z+z_{0}, d\right), V_{2}^{-}\left(z+z_{0}, d\right)\right)$ for some $z_{0} \in \mathbb{R}$.

Proposition 2.2. For each fixed $d>0$ and $c>0$, let $(U(z), V(z))$ be a solution of (2.26), then

$$
\begin{aligned}
& U(z)+V(z)-1>0 \text { for } z>-\infty, \text { if } 0<d<1 \\
& U(z)+V(z)-1 \equiv 0 \text { for } z>-\infty, \text { if } d=1 \\
& U(z)+V(z)-1<0 \text { for } z>-\infty, \text { if } d>1
\end{aligned}
$$

Proof. Let $W(z)=U(z)+V(z)-1$, then $W(z)$ satisfies

$$
W^{\prime}=-\frac{c}{d} W+\delta_{d} V^{\prime} \text { for } z \in \mathbb{R} \text {, and } W(-\infty)=0 .
$$

Thus we have $W(z)=\frac{d-1}{d} \int_{-\infty}^{z} \mathrm{e}^{\frac{c}{d}(t-z)} V^{\prime}(t) d t$. This, together with $V^{\prime}<0$, imply $(2.28)$ holds for any $d>0$.

Let $S=1-V_{1}$ be an independent variable and define $P(S)=S_{z}$ and $Q(S)=U$, then system $(2.27)$ is equivalent to the following initial value problem

$$
\left\{\begin{array}{l}
d P Q^{\prime}=P-c Q+c S, \quad S>0 \\
P P^{\prime}=-c P+Q f(1-S), \quad S>0 \\
P(0)=0, Q(0)=0, \\
P(S), Q(S)>0 \text { for } S>0
\end{array}\right.
$$


thus for each fixed $c>0$ and $d>0$ there exists a unique local positive solution $(P(S), Q(S))$ of $(2.29)$.

Note that system (2.1)-(2.2) is equivalent to the system

$$
\left\{\begin{array}{l}
d P Q^{\prime}=P-c Q+c S, \quad S \in(0,1) \\
P P^{\prime}=-c P+Q f(1-S), \quad S \in(0,1) \\
P(0)=0, Q(0)=0, \quad P(1)=0, Q(1)=1 \\
P(S), Q(S)>0 \text { for } S \in(0,1)
\end{array}\right.
$$

Lemma 2.3. Assume either (1.7) or (1.10) holds, then for each fixed $c>0$ and $d>0$ there exists a unique solution $(Q(S), P(S))$ of (2.29) satisfying

$$
P(S)=\sigma_{1}(d, c) S+o(S) \text { and } Q(S)=\sigma_{2}(d, c) S+o(S) \text { as } S \downarrow 0 .
$$

Furthermore, $P(S), Q^{\prime}(S)>0$ for all $S \in(0,1)$, and there are only two possible cases:

(a) $P(1)>0$ : there does not exist travelling wave of (2.1)-(2.2),

(b) $P(1)=0$ : there exists a travelling wave of (2.1)-(2.2).

Proof. (2.31) can be obtained by the standard asymptotic analysis on the corresponding ODE system (2.27) and phase plane analysis, only note that

$$
\mathrm{e}^{-\sigma_{1}(d, c) z}\left(U(z),-S(z),-S^{\prime}(z)\right) \rightarrow-k Y_{1}^{-} \text {as } z \rightarrow \infty, \text { for some } k>0,
$$

thus $\left(P^{\prime}(0), Q^{\prime}(0)\right)=\left(\sigma_{1}(d, c), \sigma_{2}(d, c)\right)$.

The remaining part of Lemma 2.3 can be proved by a similar argument as in [9], and so here we omit the details.

By virtue of Lemma 2.3, for any $c>0$ the unique positive local solution of (2.29) can be defined for all $S \in(0,1)$, thus we can rewrite $(2.29)$ as

$$
\left\{\begin{array}{l}
P^{\prime}=-c+\frac{Q f(1-S)}{P}, \quad S \in(0,1), \\
d P Q^{\prime}=P-c Q+c S, \quad S \in(0,1) \\
P(0)=0, Q(0)=0, \\
P(S), Q(S)>0 \text { for } S \in(0,1) .
\end{array}\right.
$$

Lemma 2.4. (i) Assume (1.10) holds, then for any $c \geq c_{*}^{0}$ and $d>1$, system (2.1)-(2.2) has a unique solution $\left(U_{c}(z), V_{c}(z)\right)$, which decays non-exponentially as $z \rightarrow+\infty$.

(ii) Assume (1.7) holds, then for any $c>c_{*}^{0}$ and $d>1$, system (2.1)-(2.2) has a unique solution $\left(U_{c}(z), V_{c}(z)\right)$, which decay exponentially with the slower exponential rate $\sigma_{+}^{+}(c)=\left(-c+\sqrt{c^{2}-4 f^{\prime}(0)}\right) / 2$ as $z \rightarrow+\infty$.

Proof. For each fixed $c \geq c_{*}^{0}$ let $\left(P_{1}(S), Q_{1}(S)\right)$ denote the unique solution of $(2.32)$ with $d=1$. Note that $Q_{1}(S) \equiv S$, and $P_{1}(S)$ satisfies

$$
\begin{aligned}
& P_{1}^{\prime}(S)=-c+\frac{S f(1-S)}{P_{1}(S)}, \quad S \in(0,1), \\
& P_{1}(0)=P_{1}(1)=0 ; \quad P_{1}(S)>0 \text { for } S \in(0,1), \\
& P^{\prime}(0)=\sigma_{1}(1, c) .
\end{aligned}
$$

For $d>1$ and a fixed $c \geq c_{*}^{0}$, let $(P(S, d), Q(S, d))$ be the unique solution of $(2.32)$, and note that $Q(S, d)<S$ for $S \in(0,1)$. By $(2.31)$ and using the fact that $\sigma_{1}(d, c)<\sigma_{1}(1, c)$ for $d>1$, there exists small $\varepsilon>0$ such that

$$
0<P(S, d)<P_{1}(S) \text { for } S \in(0, \varepsilon) \text {. }
$$


We shall first prove that

$$
0<P(S, d)<P_{1}(S) \text { for } S \in(0,1) .
$$

By contradiction, assume (2.35) does not hold, then by (2.34) there exists $B \in(0,1)$ such that

$$
0<P(S, d)<P_{1}(S) \text { for } S \in(0, B) ; \text { and } P(B, d)=P_{1}(B)>0
$$

thus $P^{\prime}(B, d) \geq P_{1}^{\prime}(B)$.

By (2.32), (2.33) and using the fact $Q(S, d)<S$ for $S \in(0,1)$ and $d>1$, we have

$$
P^{\prime}(B, d)-P_{1}^{\prime}(B)=\frac{Q(B, d)-B}{P_{*}(B)}<0,
$$

which contradicts with $P^{\prime}(B, d) \geq P_{1}^{\prime}(B)$. This completes the proof of (2.35).

(2.35) and Lemma 2.3 further imply $P(1, d)=0$ and $P^{\prime}(1, d) \geq P_{1}^{\prime}(1)$. Thus for any $c \geq c_{*}^{0}$ and $d>1$ there exists a traveling front $\left(U_{c}(z), V_{c}(z)\right)$ satisfying $(2.1)$ and $(2.2)$. Also note that $P(1, d)=0$ and $Q(1, d)=1$. This, together with the first equation of $(2.32)$, implies that $P^{\prime}(1, d)=\sigma_{+}^{-}(c)$ or $\sigma_{+}^{+}(c)$, where $\sigma_{+}^{ \pm}(c)=\left(-c \pm \sqrt{c^{2}-4 f^{\prime}(0)}\right) / 2$.

Note that if $(1.10)$ holds then $\sigma_{+}^{+}(c)=0$ and $\sigma_{+}^{-}(c)=-c$; while $\sigma_{+}^{-}(c)<\sigma_{+}^{+}(c)<0$ for $c>c_{*}^{0}$ if $(1.7)$ holds. To complete the proof of Lemma 2.4, it suffices to prove that $P^{\prime}(1, d)=\sigma_{+}^{+}(c)$.

Using the fact that $\sigma_{+}^{+}(c)$ and $\sigma_{+}^{-}(c)$ are independent of $d, P_{1}^{\prime}(1)=\sigma_{+}^{+}(c)$ for $c>c_{*}^{0}$, and $P_{1}^{\prime}(1)=\sigma_{+}^{-}(c)$ for $c=c_{*}^{0}$, which with $P^{\prime}(1, d) \geq P_{1}^{\prime}(1)$ for $d>1$ imply that

$$
P^{\prime}(1, d)=\sigma_{+}^{+}(c), \text { if } c>c_{*}^{0} ; P^{\prime}(1, d)=\sigma_{+}^{+}(c) \text { or } \sigma_{+}^{-}(c), \text { if } c=c_{*}^{0} .
$$

Finally, we shall prove

$$
P^{\prime}(1, d)=\sigma_{+}^{+}(c)=0, \text { for } d>1 \text { and } c=c_{*}^{0} \text {, if (1.10) holds. }
$$

By contradiction, assume $P^{\prime}(1, d)=\sigma_{+}^{-}(c)=-c$ for $d>1$ and $c=c_{*}^{0}$.

By (2.32) and (2.33), we have

$$
P^{\prime}(S, d)-P_{1}^{\prime}(S)+\left(\frac{S f(1-S)}{P_{1}(S) P(S, d)}\right)\left(P(S, d)-P_{1}(S)\right)=\frac{(Q-S)}{P} f(1-S)<0, \quad S \in(0,1),
$$

thus we have

$$
\left[\mathrm{e}^{\int_{1 / 2}^{S} a(z, d) d z}\left(P(S, d)-P_{1}(S)\right)\right]^{\prime}=\mathrm{e}^{\int_{1 / 2}^{S} a(z, d) d z} \frac{(Q-S)}{P} f(1-S)<0, \quad S \in(1 / 2,1),
$$

with $a(z, d)=z f(1-z) /\left(P_{1}(z) P(z, d)\right)$.

Note that

$$
\begin{aligned}
& P_{1}(z), P(z, d)=c_{*}^{0}(1-z)+o(|1-z|), \\
& \text { and } f(1-z)=(1-z)^{p}+o\left(|1-z|^{p}\right) \text { with } p>1,
\end{aligned}
$$

thus $a(z, d) \sim\left(c_{*}^{0}\right)^{-2}(1-z)^{p-2}$ as $z \rightarrow 1^{-}$for $p>1$, and $\left|\int_{1 / 2}^{1} a(z, d) d z\right|<\infty$.

Integrating the both sides of (2.37) from $1 / 2$ to 1 , we have

$$
P_{1}(1 / 2)-P(1 / 2, d)=\int_{1 / 2}^{1} \mathrm{e}^{\int_{1 / 2}^{S} a(z, d) d z} \frac{(Q-S)}{P} f(1-S) d S<0, \text { if }(1.10) \text { holds }
$$

which contradicts (2.35). This completes the proof of (2.36) and the proof of Lemma 2.4. 


\subsection{The existence of center manifold and proof of Theorem 1.2}

In this subsection, we shall further investigate the precise decaying rate of the traveling waves $\left(U_{c}(z), V_{c}(z)\right)$ obtained in Lemma 2.4(i) for the case (1.10), which decay non-exponentially as $z \rightarrow+\infty$.

For each fixed $d>0$ and $c>0$, let $(U(z), V(z))$ be a solution of $(2.1)$ satisfying $(U(+\infty), V(+\infty))=$ $(1,0)$, with $U(z)$ strictly increasing and $V(z)$ strictly deceasing, then $(U(z), V(z))$ also satisfies

$$
\left\{\begin{array}{l}
d U^{\prime}=-V^{\prime}-c(U+V-1), \\
V^{\prime \prime}+c V^{\prime}+U f(V)=0, \\
U(+\infty)=1, V(+\infty)=0, \\
0<U(z)<1, \quad 0<V(z)<1, V^{\prime}(z)<0, \text { for } z<+\infty .
\end{array}\right.
$$

Let $W(z)=U(z)+V(z)-1, V_{1}(z)=V(z)$ and $V_{2}(z)=V^{\prime}(z)$, then $\left(W(z), V_{1}(z), V_{2}(z)\right)$ satisfies the following first order differential system

$$
\left\{\begin{array}{l}
W^{\prime}=\delta_{d} V_{2}-\frac{c}{d} W \\
V_{1}^{\prime}=V_{2} \\
V_{2}^{\prime}=-c V_{2}-\left(1-V_{1}+W\right) f\left(V_{1}\right) \\
0<V_{1}(z)<1, V_{2}(z)<0
\end{array}\right.
$$

with $\delta_{d}=(d-1) / d$ and the boundary condition

$$
W(+\infty)=0, \quad V_{1}(+\infty)=0, \quad V_{2}(+\infty)=0 .
$$

Theorem 2.5. Assume (1.10) holds for some $p>1$, and let $d>0$ and $c>0$ be fixed.

(i) There exists a two-dimensional solution set $S_{0}^{+}$on which all the solutions of (2.39) decay to zero exponentially as $z \rightarrow+\infty$.

(ii) If $\left|\left(W\left(z_{0}\right), V_{1}\left(z_{0}\right), V_{2}\left(z_{0}\right)\right)\right|$ is small enough for some $z_{0} \in \mathbb{R}$ with $V_{1}\left(z_{0}\right)+V_{2}\left(z_{0}\right) / c>0$ and $\left(W\left(z_{0}\right), V_{1}\left(z_{0}\right), V_{2}\left(z_{0}\right)\right) \notin S_{0}^{+}$, then the solution $\left(W(z), V_{1}(z), V_{2}(z)\right)$ of (2.39) tends to zero algebraically as $z \rightarrow+\infty$ and satisfies

$$
\left\{\begin{array}{l}
V_{1}(z) \sim k z^{-\frac{1}{p-1}}, V_{2}(z) \sim-\frac{1}{c} k^{p} z^{-\frac{p}{p-1}}, W(z) \sim-\frac{1-d}{c^{2}} z^{-\frac{p}{p-1}} \quad \text { as } z \rightarrow+\infty, \\
\text { with } k=\left(\frac{p-1}{c}\right)^{\frac{1}{1-p}} .
\end{array}\right.
$$

Proof. By virtue of (1.10) with $p>1$, linearizing the system of (2.39) around $(0,0,0)$, we have

$$
Y^{\prime}=A^{+} Y, \text { with } A^{+}=\left(\begin{array}{ccc}
-\frac{c}{d} & 0 & \delta_{d} \\
0 & 0 & 1 \\
0 & 0 & -c
\end{array}\right)
$$

Note that $A^{+}$has three eigenvalues $-c / d,-c$ and 0 , then statement (i) follows from the standard asymptotic analysis on (2.39). Here we omit the detailed proof of (i).

Let

$$
X_{1}(z)=V_{1}(z)+\frac{1}{c} V_{2}(z), \quad X_{2}(z)=W(z)+\frac{1}{c} V_{2}(z), \quad X_{3}(z)=-\frac{1}{c} V_{2}(z),
$$

or equivalently

$$
V_{1}(z)=X_{1}(z)+X_{3}(z), V_{2}(z)=-c X_{3}(z), W(z)=X_{2}(z)+X_{3}(z),
$$

then $\left(X_{1}(z), X_{2}(z), X_{3}(z)\right)$ satisfies

$$
\left\{\begin{array}{l}
X_{1}^{\prime}=F\left(X_{1}, X_{2}, X_{3}\right) \\
X_{2}^{\prime}=-\frac{c}{d} X_{2}+F\left(X_{1}, X_{2}, X_{3}\right), \\
X_{3}^{\prime}=-c X_{3}-F\left(X_{1}, X_{2}, X_{3}\right)
\end{array}\right.
$$


with $F\left(X_{1}, X_{2}, X_{3}\right)=-\frac{1}{c}\left(1-X_{1}+X_{2}\right) f\left(X_{1}+X_{3}\right)$.

For $X_{1}>0$ small enough, let $g_{2}\left(X_{1}\right)=-\frac{d}{c^{2}} X_{1}^{p}$ and $g_{3}\left(X_{1}\right)=\frac{1}{c^{2}} X_{1}^{p}$, using (1.10) for $k_{0}=1$ and $p>1$, it can be checked that

$$
\begin{aligned}
& g_{2}^{\prime}\left(X_{1}\right) F\left(X_{1}, g_{2}\left(X_{1}\right), g_{3}\left(X_{1}\right)\right)-\left(-\frac{c}{d} g_{2}\left(X_{1}\right)+F\left(X_{1}, g_{2}\left(X_{1}\right), g_{3}\left(X_{1}\right)\right)\right. \\
& =-\frac{d p}{c^{2}} X_{1}^{p-1} \times O\left(X_{1}^{p}\right)+\frac{c}{d}\left(-\frac{d}{c^{2}} X_{1}^{p}\right)+\frac{1}{c} X_{1}^{p}+O\left(\left|X_{1}\right|^{p+1}\right) \\
& =O\left(\left|X_{1}\right|^{p+q}\right), \quad q=\min \{1, p-1\}>0,
\end{aligned}
$$

and

$$
\begin{aligned}
& g_{3}^{\prime}\left(X_{1}\right) F\left(X_{1}, g_{2}\left(X_{1}\right), g_{3}\left(X_{1}\right)\right)-\left(-c g_{3}\left(X_{1}\right)-F\left(X_{1}, g_{2}\left(X_{1}\right), g_{3}\left(X_{1}\right)\right)\right. \\
& =\frac{p}{c^{2}} X_{1}^{p-1} \times O\left(\left|X_{1}\right|^{p}\right)+\frac{1}{c} X_{1}^{p}-\frac{1}{c} X_{1}^{p}+O\left(\left|X_{1}\right|^{p+1}\right)=O\left(\left|X_{1}\right|^{p+q}\right), \quad q=\min \{1, p-1\}>0 .
\end{aligned}
$$

By applying center manifold theorems ( see [8] Theorem 3 in Chapter 2), it follows that there exists a one-dimensional center manifold $X_{2}=h_{2}\left(X_{1}\right), X_{3}=h_{3}\left(X_{1}\right)$ of (2.44) satisfying

$$
\begin{array}{r}
h_{2}\left(X_{1}\right)=-\frac{d}{c^{2}} X_{1}^{p}+O\left(\left|X_{1}\right|^{p+q}\right), h_{3}\left(X_{1}\right)=\frac{1}{c^{2}} X_{1}^{p}+O\left(\left|X_{1}\right|^{p+q}\right), \text { for small enough } X_{1} \geq 0, \\
q=\min \{1, p-1\}>0 ;
\end{array}
$$

thus the flow of (2.44) on the center manifold $X_{2}=h_{2}\left(X_{1}\right), X_{3}=h_{3}\left(X_{1}\right)$ with $X_{1} \geq 0$ is governed by the following scalar equation

$$
u^{\prime}=F\left(u, h_{2}(u), h_{3}(u)\right)=-\frac{1}{c} f(u)+O\left(|u|^{p+1}\right), \text { for small enough } u \geq 0 .
$$

Obviously for $p>1$ and $c>0$, the zero solution of (2.48) is locally asymptotically stable and it is easy to prove that if $u(0)>0$ is small enough then the solution of (2.48) satisfies

$$
u(z)>0 \text { for } z \geq 0 \text {, and } u(z)=\left(\frac{p-1}{c}\right)^{\frac{1}{1-p}} z^{-\frac{1}{p-1}}+o\left(z^{-\frac{1}{p-1}}\right) \text { as } z \rightarrow+\infty .
$$

By virtue of (2.47)-(2.48), the center manifold theories (see [8] Theorem 2 in Chapter 2) also guarantee that the zero solution of (2.44) is also locally asymptotically stable for $X_{1}(0)>0$; furthermore, for each fixed small $\left(X_{1}(0), X_{2}(0), X_{3}(0)\right)$ with $X_{1}(0)>0$, there exists a solution $u(z) \geq 0$ of $(2.48)$ such that the solution $\left(X_{1}(z), X_{2}(z), X_{3}(z)\right)$ of (2.44) satisfies

$$
\left\{\begin{array}{l}
X_{1}(z)=u(z)+O\left(\mathrm{e}^{-\gamma z}\right) \\
X_{2}(z)=h_{2}(u(z))+O\left(\mathrm{e}^{-\gamma z}\right), \gamma=\min \{c, c / d\}, \quad \text { as } z \rightarrow+\infty, \\
X_{3}(z)=h_{3}(u(z))+O\left(\mathrm{e}^{-\gamma z}\right),
\end{array}\right.
$$

which with (2.47) and (2.49) further imply that if $\left(X_{1}(0), X_{2}(0), X_{3}(0)\right)$ is small enough with $X_{1}(0)>$ 0 , then the solution $\left(X_{1}(z), X_{2}(z), X_{3}(z)\right)$ of (2.44) either decay exponentially as $z \rightarrow+\infty$ or decay algebraically satisfying

$$
X_{1}(z) \sim k z^{-\frac{1}{p-1}}, X_{2}(z) \sim-\frac{d}{c^{2}} k^{p} z^{-\frac{p}{p-1}}, X_{3}(z) \sim \frac{1}{c^{2}} k^{p} z^{-\frac{p}{p-1}}, \text { as } z \rightarrow \infty, \text { with } k=\left(\frac{p-1}{c}\right)^{\frac{1}{1-p}} .
$$

(2.50) also imply that except on the two-dimensional solution set $S^{+}$, all the solutions of (2.44) near zero with $X_{1}(z)>0$ satisfy (2.51) as $z \rightarrow+\infty$, which with (2.43) further imply the estimates (ii) of Theorem 2.5 hold. This completes the proof of Theorem 2.5.

Proof of Theorem 1.2. (i) First for the case $0<d<1$, using the fact $c_{*}(d)=c_{\min }(d)$ and the monotone decreasing of $c_{\min }(d)$ in $d$ (see Theorem 1), we have $c_{*}(d) \geq c_{*}^{0}$.

For the case $d>1$, estimate $c_{*}(d) \leq c_{*}^{0}$ follows from Lemma 2.4 directly.

(ii) For the case $0<d<1$, the existence and decaying estimates of the waves with noncritical speeds $c>c_{*}(d)$ stated in Theorem 1.2 follow from Theorem 1 (ii) and Theorem 2.5.

For the case $d>1$, the results stated in (ii) follow from Lemma 2.4 and Theorem 2.5. 


\section{Linear stability of wave fronts with exponential decay for $d$ near 1}

Under the assumption of $(1.7)$ or $(1.10)$, let $\left(U_{c}(x-c t, d), V_{c}(x-c t, d)\right)$ be a traveling front solution obtained in Theorem 1.1 and Theorem 1.2 for $d$ near 1 . In this section we shall investigate the spectral and linear stability of the wave front in some suitable spaces.

In moving coordinate $z=x-c t$ the original nonlinear systems (1.1) becomes

$$
\left\{\begin{array}{l}
u_{t}=d u_{z z}+c u_{z}-u f(v) \\
v_{t}=v_{z z}+c v_{z}+u f(v)
\end{array}\right.
$$

Linearizing system $(3.1)$ around $\left(U_{c}(z), V_{c}(z)\right)$ we can define the linear operator $\mathcal{L}_{d, c}$ : $C_{\text {unif }}^{2}(\mathbb{R}) \times$ $C_{\text {unif }}^{2}(\mathbb{R}) \rightarrow C_{\text {unif }}(\mathbb{R}) \times C_{\text {unif }}(\mathbb{R})$ by

$$
\mathcal{L}_{d, c}=\left(\begin{array}{cc}
d \frac{\partial^{2}}{\partial z^{2}}+c \frac{\partial}{\partial z}-f\left(V_{c}\right) & -U_{c} f^{\prime}\left(V_{c}\right) \\
f\left(V_{c}\right) & \frac{\partial^{2}}{\partial z^{2}}+c \frac{\partial}{\partial z}+U_{c} f^{\prime}\left(V_{c}\right)
\end{array}\right) .
$$

Let $(u(z, t), v(z, t))$ be a solution of the nonlinear system (3.1), and define $w=u+v-1$, then $(w(z, t), v(z, t))$ satisfies

$$
\left\{\begin{array}{l}
w_{t}=d w_{z z}+(1-d) v_{z z}+c w_{z} \\
v_{t}=v_{z z}+c v_{z}+(1-v+w) f(v),
\end{array}, z \in \mathbb{R}, t>0\right.
$$

Obviously, $\left(W_{c}(z, d), V_{c}(z, d)\right)=\left(U_{c}(z, d)+V_{c}(z, d)-1, V_{c}(z, d)\right)$ is a steady state solution of (3.3), the linearized system of $(3.3)$ around $\left(W_{c}(z, d), V_{c}(z, d)\right)$ is the following

$$
\left\{\begin{array}{l}
w_{t}=d w_{z z}+(1-d) v_{z z}+c w_{z} \\
v_{t}=v_{z z}+c v_{z}+F_{v}\left(W_{c}, V_{c}\right) v+F_{w}\left(W_{c}, V_{c}\right) w,
\end{array}, z \in \mathbb{R}, t>0\right.
$$

with $F(w, v)=(1-v+w) f(v)$.

Define the linear operator $\widetilde{\mathcal{L}}_{d, c}: C_{\text {unif }}^{2}(\mathbb{R}) \times C_{\text {unif }}^{2}(\mathbb{R}) \rightarrow C_{\text {unif }}(\mathbb{R}) \times C_{\text {unif }}(\mathbb{R})$ by

$$
\widetilde{\mathcal{L}}_{d, c}=\left(\begin{array}{cc}
d \frac{\partial^{2}}{\partial z^{2}}+c \frac{\partial}{\partial z} & (1-d) \frac{\partial^{2}}{\partial z^{2}} \\
F_{w}\left(W_{c}, V_{c}\right) & \frac{\partial^{2}}{\partial z^{2}}+c \frac{\partial}{\partial z}+F_{v}\left(W_{c}, V_{c}\right)
\end{array}\right) .
$$

It is obvious that for each fixed $d>0$, both $\mathcal{L}_{d, c}$ and $\widetilde{\mathcal{L}}_{d, c}$ generate analytic semigroups on $X=C_{\text {unif }}(\mathbb{R}) \times$ $C_{\text {unif }}(\mathbb{R})$. For any given pair $\alpha=\left(\alpha^{-}, \alpha^{+}\right)$with $\alpha^{-} \geq 0$ and $\alpha^{+}>0$, it is easy to check that $\mathcal{L}_{d, c}$ and $\widetilde{\mathcal{L}}_{d, c}$ also generate analytic semigroups on the exponentially weighted space $X_{\alpha}=C_{\alpha}(\mathbb{R}) \times C_{\alpha}(\mathbb{R})$, with $C_{\alpha}(\mathbb{R}) \triangleq\left\{u \in C(\mathbb{R}) \mid w_{\alpha} u(z) \in C_{\text {unif }}(\mathbb{R})\right\}$ and $w_{\alpha}(z)$ defined as in (1.16); the weighted spaces $C_{\alpha}^{2}(\mathbb{R})$ and $X_{\alpha}^{2}$ can be defined similarly.

Define operators $\mathcal{L}_{d, c, \alpha}, \widetilde{\mathcal{L}}_{d, c, \alpha}: X_{\alpha}^{2} \rightarrow X_{\alpha}$ by

$$
\mathcal{L}_{d, c, \alpha}\left(\begin{array}{l}
u \\
v
\end{array}\right)=\mathcal{L}_{d, c}\left(\begin{array}{l}
u \\
v
\end{array}\right) \text { for }\left(\begin{array}{l}
u \\
v
\end{array}\right) \in X_{\alpha}^{2},
$$

and

$$
\widetilde{\mathcal{L}}_{d, c, \alpha}\left(\begin{array}{l}
w \\
v
\end{array}\right)=\widetilde{\mathcal{L}}_{d, c}\left(\begin{array}{l}
w \\
v
\end{array}\right) \text { for }\left(\begin{array}{l}
w \\
v
\end{array}\right) \in X_{\alpha}^{2} .
$$

It is easy to see that the linear operator $\mathcal{L}_{d, c}$ is equivalent to $\widetilde{\mathcal{L}}_{d, c}$ in the sense that

$$
\sigma\left(\mathcal{L}_{d, c}\right)=\sigma\left(\widetilde{\mathcal{L}}_{d, c}\right) \text { and } \sigma\left(\mathcal{L}_{d, c, \alpha}\right)=\sigma\left(\widetilde{\mathcal{L}}_{d, c, \alpha}\right) .
$$


Consider the eigenvalue problem of $\widetilde{\mathcal{L}}_{d, c}$, i.e.

$$
\widetilde{\mathcal{L}}_{d, c}\left(\begin{array}{l}
w \\
v
\end{array}\right)=\lambda\left(\begin{array}{l}
w \\
v
\end{array}\right)
$$

which can be rewritten as the following first order differential system

$$
Y^{\prime}(z)=A(z, \lambda, d, c) Y(z)
$$

with $Y(z)=\left(w(z), w^{\prime}(z), v(z), v^{\prime}(z)\right)^{T} \triangleq\left(y_{1}, y_{2}, y_{3}, y_{4}\right)^{T}$, and

$$
A(z, \lambda, d, c)=\left(\begin{array}{cccc}
0 & 1 & 0 & 0 \\
\frac{\lambda}{d}-a_{1}(z, d, c) \delta_{d} & \frac{-c}{d} & -a_{2}(z, d, c) \delta_{d}+\lambda \delta_{d} & -c \delta_{d} \\
0 & 0 & 0 & 1 \\
-a_{1}(z, d, c) & 0 & \lambda-a_{2}(z, d, c) & -c
\end{array}\right)
$$

where $\delta_{d}=\frac{d-1}{d}, a_{1}(z, d, c)=F_{w}\left(W_{c}, V_{c}\right)=f\left(V_{c}\right)$ and $a_{2}(z, d, c)=F_{v}\left(W_{c}, V_{c}\right)=\left(1+W_{c}-V_{c}\right) f^{\prime}\left(V_{c}\right)-$ $f\left(V_{c}\right)$.

Note that

$$
a_{1}(z, d, c) \rightarrow 0, \quad a_{2}(z, d, c) \rightarrow f^{\prime}(0) \text { as } z \rightarrow+\infty
$$

and

$$
a_{1}(z, d, c) \rightarrow 1, \quad a_{2}(z, d, c) \rightarrow-1 \text { as } z \rightarrow-\infty
$$

then

$$
A(+\infty, \lambda, d, c)=\left(\begin{array}{cccc}
0 & 1 & 0 & 0 \\
\frac{\lambda}{d} & \frac{-c}{d} & -f^{\prime}(0) \delta_{d}+\lambda \delta_{d} & -c \delta_{d} \\
0 & 0 & 0 & 1 \\
0 & 0 & \lambda-f^{\prime}(0) & -c
\end{array}\right)
$$

and

$$
A(-\infty, \lambda, d, c)=\left(\begin{array}{cccc}
0 & 1 & 0 & 0 \\
\frac{\lambda}{d}-\delta_{d} & \frac{-c}{d} & \lambda \delta_{d}+\delta_{d} & -c \delta_{d} \\
0 & 0 & 0 & 1 \\
-1 & 0 & \lambda+1 & -c
\end{array}\right)
$$

Denote the four eigenvalues of $A(+\infty, \lambda, d, c)$ by $\mu_{i}^{+}(\lambda, d, c)(i=1, \ldots, 4)$ with

$$
\begin{cases}\mu_{1}^{+}(\lambda, d, c)=\frac{-c-\sqrt{c^{2}+4 \lambda-4 f^{\prime}(0)}}{2}, & \mu_{2}^{+}(\lambda, d, c)=\frac{-c-\sqrt{c^{2}+4 \lambda d}}{2 d} \\ \mu_{3}^{+}(\lambda, d, c)=\frac{-c+\sqrt{c^{2}+4 \lambda-4 f^{\prime}(0)}}{2}, & \mu_{4}^{+}(\lambda, d, c)=\frac{-c+\sqrt{c^{2}+4 \lambda d}}{2 d}\end{cases}
$$

It is easy to check that for each fixed $c \geq c_{*}(d)>0$ and $d>0$, it holds that

$$
\operatorname{Re} \mu_{1}^{+}(\lambda, d, c), \operatorname{Re} \mu_{2}^{+}(\lambda, d, c) \leq \max \left\{\frac{-c-\sqrt{c^{2}-4 f^{\prime}(0)}}{2},-c / d\right\} \text { for } \operatorname{Re} \lambda \geq 0 ;
$$

$\left\{\begin{array}{l}\operatorname{Re} \mu_{3}^{+}(\lambda, d, c), \operatorname{Re} \mu_{4}^{+}(\lambda, d, c)>0, \quad \text { if }(1.10) \text { holds, } \\ \operatorname{Re} \mu_{3}^{+}(\lambda, d, c)>\frac{-c+\sqrt{c^{2}-4 f^{\prime}(0)}}{2}, \operatorname{Re} \mu_{4}^{+}(\lambda, d, c)>0, \text { if (1.7) holds, for } \operatorname{Re} \lambda \geq 0 \text { and } \lambda \neq 0\end{array}\right.$

and

$$
\mu_{3}^{+}(0, d, c)=\mu_{4}^{+}(0, d, c)=0, \text { if }(1.10) \text { holds }
$$


It can be verified that if $c>2 \sqrt{f^{\prime}(0)}$ then for each fixed $\alpha^{+} \in\left(\left(c-\sqrt{c^{2}-4 f^{\prime}(0)}\right) / 2, c / 2\right)$ there exists small $\delta_{\alpha}>0$, such that

$$
\begin{array}{r}
\operatorname{Re} \mu_{1}^{+}(\lambda, d, c), \operatorname{Re} \mu_{2}^{+}(\lambda, d, c)<-c / 2<-\alpha^{+}<\operatorname{Re} \mu_{3}^{+}(\lambda, d, c), \operatorname{Re} \mu_{4}^{+}(\lambda, d, c), \\
\text { for } \operatorname{Re} \lambda>-\delta_{\alpha} \text { and }|d-1| \leq \delta_{\alpha} .
\end{array}
$$

It can be checked that for $d=1$ and $c>0, A(-\infty, \lambda, d, c)$ has four distinct eigenvalues denoted by $\mu_{i}^{-}(\lambda, 1)$ with

$$
\left\{\begin{array}{l}
\mu_{1}^{-}(\lambda, 1, c)=\frac{-c-\sqrt{c^{2}+4(\lambda+1)}}{2}, \quad \mu_{2}^{-}(\lambda, 1, c)=\frac{-c-\sqrt{c^{2}+4 \lambda}}{2} \\
\mu_{3}^{-}(\lambda, 1, c)=\frac{-c+\sqrt{c^{2}+4 \lambda}}{2}, \quad \mu_{4}^{-}(\lambda, 1, c)=\frac{-c+\sqrt{c^{2}+4(\lambda+1)}}{2}
\end{array}\right.
$$

and for each fixed $c>0$ and small $\alpha^{-}>0$ if necessary, we choose $\delta_{\alpha}>0$ smaller such that

$$
\begin{aligned}
& \operatorname{Re} \mu_{1}^{-}(\lambda, 1, c), \operatorname{Re} \mu_{2}^{-}(\lambda, 1, c)<-\frac{3 c}{4}<-\alpha^{-}<\operatorname{Re} \mu_{3}^{-}(\lambda, 1, c), \operatorname{Re} \mu_{4}^{-}(\lambda, 1, c) \\
& \text { for } \operatorname{Re} \lambda>-\delta_{\alpha} .
\end{aligned}
$$

Then by applying the classical perturbation theories of eigenvalues (see [17]), it follows that for each fixed small $\alpha^{-} \in(0, c / 2)$ and bounded $\Lambda>0$, there exists small $\delta_{\alpha, \Lambda}>0$ such that for any $\operatorname{Re} \lambda>$ $-\delta_{\alpha, \Lambda},|\lambda|<\Lambda$ and $|d-1|<\delta_{\alpha, \Lambda}, A(-\infty, \lambda, d, c)$ has four distinct eigenvalues denoted by $\mu_{i}^{-}(\lambda, d, c)$, which are analytic in $\lambda$ and continuous in $d$ and satisfy

$$
\left\{\begin{array}{l}
\operatorname{Re} \mu_{1}^{-}(\lambda, d, c)<\operatorname{Re} \mu_{2}^{-}(\lambda, d, c)<-\frac{c}{2}<-\alpha^{-}<\operatorname{Re} \mu_{3}^{-}(\lambda, d, c)<\operatorname{Re} \mu_{4}^{-}(\lambda, d, c) \\
\quad \text { for } \operatorname{Re} \lambda>-\delta_{\alpha, \Lambda},|\lambda|<\Lambda \text { and }|d-1|<\delta_{\alpha, \Lambda},
\end{array}\right.
$$

with four corresponding linearly independent eigenvectors denoted by $V_{i}^{-}(\lambda, d)$, which are also analytic in $\lambda$ and continuous in $d$.

Define curves $S^{+}(d, c)$ and $S^{-}(d, c)$ by

$$
\begin{gathered}
S_{d}^{+}=\{\lambda \in \mathbb{C} \mid \operatorname{det}(\mathrm{i} \tau I-A(+\infty, \lambda, d, c))=0 \text { for some } \tau \in \mathbb{R}\} \text { and } \\
S_{d}^{-}=\{\lambda \in \mathbb{C} \mid \operatorname{det}(\mathrm{i} \tau I-A(-\infty, \lambda, d, c))=0 \text { for some } \tau \in \mathbb{R}\} .
\end{gathered}
$$

By applying the spectral theory in [14], the boundary of essential spectrum of $\widetilde{\mathcal{L}}_{d, c}$ is characterized by $S_{d}^{+}$ and $S_{d}^{-}$, and $S_{d}^{+}, S_{d}^{-} \subset \sigma_{\text {ess }}\left(\widetilde{\mathcal{L}}_{d}\right)$, then by detailed computation or by (3.12)-(3.15) and (3.17), it follows that

Lemma 3.1. (i) If (1.10) holds, then for any $d>0 \operatorname{Re} \sigma_{\text {ess }}\left(\widetilde{\mathcal{L}}_{d, c}\right) \leq 0$, and $0 \in \sigma_{\text {ess }}\left(\widetilde{\mathcal{L}}_{d, c}\right)$. (ii) If (1.7) holds and $c_{*}(d) \geq 2 \sqrt{f^{\prime}(0)}$, then for small $|d-1| \quad \sigma_{\mathrm{ess}}\left(\widetilde{\mathcal{L}}_{d, c}\right) \cap\{\operatorname{Re} \lambda \geq 0\} \neq \emptyset$.

Lemma 3.1 implies that all the waves with critical or noncritical speeds can not be exponentially stable in space $X$. To get the linear exponential stability of the waves in some appropriate spaces, we first investigate the location of essential spectrum of $\mathcal{L}_{d, c}$ in some exponentially weighted space of $X$.

For the fixed pair $\alpha=\left(\alpha^{-}, \alpha^{+}\right)$with $\alpha^{-}, \alpha^{+}>0$, define the weight function $w_{\alpha}(z) \in C^{2}(\mathbb{R})$ as in (1.16), and define operator $\widehat{\mathcal{L}}_{d, c, \alpha}: C_{\text {unif }}^{2}(\mathbb{R}) \times C_{\text {unif }}^{2}(\mathbb{R}) \rightarrow C_{\text {unif }}(\mathbb{R}) \times C_{\text {unif }}(\mathbb{R})$ by

$$
\widehat{\mathcal{L}}_{d, c, \alpha}\left(\begin{array}{c}
w(z) \\
v(z)
\end{array}\right)=w_{\alpha}^{-1}(z) \widetilde{\mathcal{L}}_{d, c}\left(\begin{array}{c}
w_{\alpha}(z) w(z) \\
w_{\alpha}(z) v(z)
\end{array}\right) .
$$

Obviously, the operator $\widehat{\mathcal{L}}_{d, c, \alpha}$ is equivalent to the operator $\widetilde{\mathcal{L}}_{d, c, \alpha}$ and operator $\mathcal{L}_{d, c, \alpha}$, thus $\sigma_{\text {ess }}\left(\widehat{\mathcal{L}}_{d, c, \alpha}\right)=$ $\sigma_{\text {ess }}\left(\widetilde{\mathcal{L}}_{d, c, \alpha}\right)=\sigma_{\text {ess }}\left(\mathcal{L}_{d, c, \alpha}\right)$. By (1.16), (3.8), (3.20) and by applying the standard spectral theories in [14], it is easy to prove that the boundary of $\sigma_{\text {ess }}\left(\widehat{\mathcal{L}}_{d, c, \alpha}\right)$ can be characterized by the curves $S_{d, c, \alpha^{-}}^{-}$and $S_{d, c, \alpha^{+}}^{+}$, which are defined by

$$
\begin{gathered}
\left.S_{d, c, \alpha^{+}}^{+}=\left\{\lambda \in \mathbb{C} \mid \operatorname{det}\left(\mathrm{i} \tau I-\alpha^{+} I-A(+\infty, \lambda, d, c)\right)\right)=0 \quad \text { for some } \tau \in \mathbb{R}\right\}, \\
\text { and } S_{d, c, \alpha^{-}}^{-}=\left\{\lambda \in \mathbb{C} \mid \operatorname{det}\left(\mathrm{i} \tau I-\alpha^{-} I-A(-\infty, \lambda, d, c)\right)=0 \quad \text { for some } \tau \in \mathbb{R}\right\} .
\end{gathered}
$$

Further, by (3.16) and (3.19) or by direct computation, we have the following estimates 
Lemma 3.2. Assume either (1.7) or (1.10) holds, then for $c>2 \sqrt{f^{\prime}(0)}, \alpha^{-} \in(0, c / 2)$ and $\alpha^{+} \in$ $\left(\left(c-\sqrt{c^{2}-4 f^{\prime}(0)}\right) / 2, c / 2\right)$, there exist small positive constants $\delta_{0}$ and $\delta_{\alpha}$ such that

$$
\sup \left\{\operatorname{Re} \sigma_{\text {ess }}\left(\widetilde{\mathcal{L}}_{d, c, \alpha}\right)\right\} \leq-\delta_{\alpha}<0 \text { for any }|d-1|<\delta_{0} .
$$

Remark 3.3. If (1.7) holds and $c_{*}(d)=2 \sqrt{f^{\prime}(0)}$, then it is easy to check that for $c=c_{*}(d)$ there exists no appropriate exponential weight function $w_{\alpha}(z)$, such that $\sigma_{\mathrm{ess}}\left(\mathcal{L}_{d, c, \alpha}\right)$ can be shifted to the left of the imaginary axis of the complex plane.

Under the assumption of Theorem 1.1, for $c=c_{*}^{0}, \alpha^{+} \in\left(\left(c_{*}^{0}-\sqrt{\left(c_{*}^{0}\right)^{2}-4 f^{\prime}(0)}\right) / 2, c_{*}^{0} / 2\right), \alpha^{-} \in\left(0, c_{*}^{0} / 4\right)$ and small $\delta_{0}>0$, let $\lambda$ with $\operatorname{Re} \lambda \geq-\delta_{0}$ be an eigenvalue of $\widetilde{\mathcal{L}}_{1, c, \alpha}$ with eigenfunction $\left(w_{\lambda}(z), v_{\lambda}(z)\right) \in X_{\alpha}$ satisfying

$$
\left\{\begin{array}{l}
w_{\lambda}^{\prime \prime}+c w_{\lambda}^{\prime}=\lambda w_{\lambda} \\
v_{\lambda}^{\prime \prime}+c v_{\lambda}^{\prime}+F_{v}\left(0, V_{c}\right) v_{\lambda}+F_{w}\left(0, V_{c}\right) w_{\lambda}=\lambda v_{\lambda} .
\end{array}\right.
$$

From the first equation of (3.21), it is easy to prove that if $\left(w_{\lambda}(z), v_{\lambda}(z)\right) \in X_{\alpha}$ is a solution of (3.21) for $\operatorname{Re} \lambda \geq-\delta_{\alpha}$, then $w_{\lambda}(z) \equiv 0$ and $v_{\lambda}(z) \in C_{\alpha}(\mathbb{R})$ satisfies

$$
\lambda v_{\lambda}=v_{\lambda}^{\prime \prime}+c_{*}^{0} v_{\lambda}^{\prime}+F_{v}\left(0, V_{c}\right) v \triangleq L v_{\lambda} .
$$

By applying standard asymptotic analysis to the eigenvalue problem (3.21) and by Lemma 2.1, it is easy to prove that

Lemma 3.4. Assume either (1.10) holds or (1.7) and $c_{*}^{0}>2 \sqrt{f^{\prime}(0)}$ holds. Let $\alpha^{-} \in\left(0, c_{*}^{0} / 4\right), \alpha^{+} \in$ $\left(\left(c_{*}^{0}-\sqrt{\left(c_{*}^{0}\right)^{2}-4 f^{\prime}(0)}\right) / 2, c_{*}^{0} / 2\right)$, then there exists small positive constant $\delta_{\alpha}>0$ such that

$$
\sup \left\{\operatorname{Re}\left\{\sigma_{p}\left(\widetilde{\mathcal{L}}_{1, c_{*}^{0}, \alpha}\right) \backslash\{0\}\right\}\right\}<-\delta_{\alpha}<0,
$$

and zero is a simple eigenvalue of $\widetilde{\mathcal{L}}_{1, c_{*}^{0}, \alpha}$.

Under the assumptions of Lemma 3.4, by virtue of Theorem 1.1, Lemma 3.2 and Lemma 3.4, for the fixed $\alpha^{-} \in\left(0, c_{*}^{0} / 4\right)$ and $\alpha^{+} \in\left(\left(c_{*}^{0}-\sqrt{\left(c_{*}^{0}\right)^{2}-4 f^{\prime}(0)}\right) / 2, c_{*}^{0} / 2\right)$, in the following we always choose $\delta_{0}>0$ and $\delta_{\alpha}>0$ small enough such that Lemma 3.4 holds and

$$
\sup \left\{\operatorname{Re} \sigma_{\text {ess }}\left(\widetilde{\mathcal{L}}_{d, c_{*}(d), \alpha}\right)\right\} \leq-\delta_{\alpha}<0 \text { for any }|d-1|<\delta_{0}
$$

Further, we shall apply Evans function method to prove the similar spectral estimates as stated in Lemma 3.4 hold true for $d$ near 1. Before applying Evans function method, we need to prove the uniform boundedness of unstable eigenvalues of $\widetilde{\mathcal{L}}_{d, c_{*}(d), \alpha}$ when $d$ is near 1. By applying similar energy estimates as in [21] (see the proof of Theorem 3.4 in [21]), we can obtain the following estimates.

Lemma 3.5. Assume either (1.10) holds or (1.7) and $c_{*}^{0}>2 \sqrt{f^{\prime}(0)}$ hold, and let $\alpha^{-}$and $\alpha^{+}$satisfy the assumption of Lemma 3.4, then there exist small $\delta_{\alpha}>0, \delta_{0}>0$ and large $\Lambda_{0}>0$ such that $\widetilde{\mathcal{L}}_{d, c_{*}(d), \alpha}$ has no eigenvalues in $\left\{\lambda \in \mathbb{C}\left|\operatorname{Re} \lambda \geq-\delta_{\alpha},\right| \lambda \mid \geq \Lambda_{0}\right\}$ if $|d-1| \leq \delta_{0}$.

Let $\alpha, \delta_{0}, \delta_{\alpha}$ and $\Lambda_{0}$ be fixed to satisfy the assumptions in Lemmas 3.2-3.5. Further, by virtue of the spectral gaps in (3.16) and (3.19) and the exponential spatial decay of the waves with speed $c_{*}(d)$ for $d$ near 1 , we can apply the standard asymptotic ODE theories and similar arguments as in [2] and [23] or apply the more general results on Evans function obtained in [21] ( in [21], $A(z, \lambda, d, c)$ is allowed to be slow algebraic decay at $z= \pm \infty$ and the eigenvalues $\mu_{i}^{ \pm}(\lambda, d, c)$ can be not simple) to prove that for small $|d-1| \leq \delta_{0}, c=c_{*}(d)$ and $\lambda \in \Omega_{0} \triangleq\left\{\lambda \in \mathbb{C}\left|\operatorname{Re} \lambda \geq-\delta_{\alpha},\right| \lambda \mid \leq \Lambda_{0}\right\}$, there exist two families of four linearly independent solutions to system (3.8) denoted by $\left\{Y_{i}^{-}(z, d, c, \lambda)\right\}$ and $\left\{Y_{i}^{-}(z, d, c, \lambda)\right\}$ $(i=1, \ldots, 4)$ satisfying

$$
\mathrm{e}^{-\mu_{i}^{-}(\lambda, d, c) z} Y_{i}^{-}(z, d, c, \lambda) \rightarrow V_{i}^{-}(\lambda, d, c), \quad i=1, \ldots, 4, \text { as } z \rightarrow-\infty
$$




$$
\mathrm{e}^{\alpha^{+} z} Y_{i}^{+}(z, d, c, \lambda) \rightarrow 0, \quad i=1,2, \text { as } z \rightarrow+\infty
$$

and

$$
\mathrm{e}^{\alpha^{+} z} Y_{i}^{+}(z, d, c, \lambda) \rightarrow+\infty, \quad i=3,4, \text { as } z \rightarrow+\infty
$$

where $Y_{i}^{-}(z, d, c, \lambda)(i=1, \ldots, 4)$, the wedges $Y_{1}^{+} \wedge Y_{2}^{+}(z, d, c, \lambda)$ and $Y_{3}^{+} \wedge Y_{4}^{+}(z, d, c, \lambda)$ are analytic in $\lambda$ and continuous in $d$ and $c$; the detailed constructive proof of the existence and analyticity of $Y_{i}^{ \pm}(z, d, c, \lambda)$ $(i=1, \ldots, 4)$ can be found in $[21]$.

By virtue of the existence, analyticity and decaying estimates of $Y_{i}^{ \pm}(z, d, c, \lambda)(i=1, \ldots, 4)$, for $\lambda \in \Omega_{0}$ and $d$ near 1 we can define Evans function $([2,21])$ by

$$
D(\lambda, d)=\exp \left\{-\int_{0}^{z} A\left(s, \lambda, d, c_{*}(d)\right) \mathrm{d} s\right\} \operatorname{det}\left[Y_{1}^{+} Y_{2}^{+} Y_{3}^{-} Y_{4}^{-}\right]\left(z, d, c_{*}(d), \lambda\right),
$$

which is independent of $z$ and analytic in $\lambda$ and continuous in $d$. Furthermore, $D(\lambda, d)=0$ if and only if $\lambda$ is an eigenvalue of $\widetilde{\mathcal{L}}_{d, c_{*}}(d), \alpha$ with an eigenfunction $\left(w_{\lambda}(z, d), v_{\lambda}(z, d)\right) \in X_{\alpha}$; and the number of the zeros (counting the algebraic multiplicities) of $D(\lambda, d)$ in $\Omega_{0}$ equates the number of eigenvalues of $\widetilde{\mathcal{L}}_{d, c_{*}}(d), \alpha$ (counting the algebraic multiplicities) in $\Omega_{0}$.

Note that Lemma 3.4 guarantees that the Evans function $D(\lambda, d)$ at $d=1$ has exactly one zero (counting the algebraic multiplicity) in $\Omega_{0}$, then by applying Rouchét Theorem it follows that for each fixed $d$ near 1 there is exactly one zero of $D(\lambda, d)$ in $\Omega_{0}$. Further, note that zero is an eigenvalue of $\widetilde{\mathcal{L}}_{d, c_{*}(d), \alpha}$ for $d$ near 1 , with eigenfunction $\left(W_{c_{*}(d)}^{\prime}(z, d), V_{c_{*}(d)}^{\prime}(z, d)\right) \in X_{\alpha}$, then it follows that $\widetilde{\mathcal{L}}_{d, c_{*}}(d), \alpha$ has no eigenvalues in $\Omega_{0}$ except at $\lambda=0$ and zero is a simple eigenvalue of $\widetilde{\mathcal{L}}_{d, c_{*}}(d), \alpha$, which with Lemma 3.5 further implies the spectral results stated in Lemma 3.4 are still valid for $\widetilde{\mathcal{L}}_{d, c_{*}}(d), \alpha$ for $d$ near 1 . Then by virtue of (3.6) and (3.23) we also have the following spectral estimates on $\mathcal{L}_{d, c_{*}(d), \alpha}$.

Lemma 3.6. Assume either (1.10) holds or (1.7) and $c_{*}^{0}>2 \sqrt{f^{\prime}(0)}$ hold. For each fixed $\alpha^{-} \in\left(0, c_{*}^{0} / 4\right)$ and $\alpha^{+} \in\left(\left(c_{*}^{0}-\sqrt{\left(c_{*}^{0}\right)^{2}-4 f^{\prime}(0)}\right) / 2, c_{*}^{0} / 2\right)$, there exist positive constants $\delta_{\alpha}$ and $\delta_{0}$ such that for any $|d-1| \leq \delta_{0}$

$$
\sup \left\{\operatorname{Re}\left\{\sigma_{p}\left(\mathcal{L}_{d, c_{*}}(d), \alpha\right) \backslash\{0\}\right\}\right\}<-\delta_{\alpha}<0,
$$

and zero is a simple eigenvalue of $\mathcal{L}_{d, c_{*}(d), \alpha}$ with eigenspace spanned by $\Phi_{0}^{d}(z)=\left(U_{c_{*}(d)}^{\prime}(z, d), V_{c_{*}(d)}^{\prime}(z, d)\right)$.

By applying the classical semigroup theories, Lemma 3.6 implies that under the assumption of Lemma 3.6 for each fixed $d$ near 1, Range $\left\{\mathcal{L}_{d, c_{*}(d), \alpha}\right\}$ is closed and we can define the Riesz spectral projection $P_{\alpha}^{d}$ of $X_{\alpha}$ onto the one-dimensional space $\operatorname{Ker}\left\{\mathcal{L}_{d, c_{*}(d), \alpha}\right\}=\operatorname{Span}\left\{\Phi_{0}^{d}(z)\right\}$, thus the weighted space $X_{\alpha}$ can be decomposed as follows

$$
X_{\alpha}=\operatorname{Ker}\left\{\mathcal{L}_{d, c_{*}(d), \alpha}\right\} \bigoplus Y_{d, \alpha}, \text { with } Y_{d, \alpha}=\left(I-P_{\alpha}^{d}\right) X_{\alpha}=\operatorname{Range}\left\{\mathcal{L}_{d, c_{*}(d), \alpha}\right\} \subset X_{\alpha} .
$$

Define a linear bounded functional $\rho: X_{\alpha} \rightarrow R$ such that

$$
P_{\alpha}^{d} Y=\rho(Y) \Phi_{0}^{d} \text { for } Y \in X_{\alpha} \text {, and } \rho\left(\Phi_{0}^{d}\right)=1 \text {. }
$$

Denote $Q_{\alpha}^{d}=I-P_{\alpha}^{d}$, which is a projection onto $Y_{d, \alpha}$. Obviously both $P_{\alpha}^{d}$ and $Q_{\alpha}^{d}$ commute with $\mathrm{e}^{t \mathcal{L}_{d, c_{*}(d), \alpha}}$ for all $t \geq 0$.

Denote $\mathcal{L}_{d, c_{*}(d), \alpha} Q_{\alpha}^{d}$ by $\mathcal{L}_{d, \alpha}^{0}$, then Lemma 3.6 and (3.25) imply that $\operatorname{Re}\left\{\mathcal{L}_{d, \alpha}^{0}\right\} \leq-\delta_{\alpha}$, which further guarantees the linear exponential stability of the wave front $\left(U_{c_{*}(d)}(z, d), V_{c_{*}(d)}(z, d)\right)$ with perturbation in the weighted space $Y_{d, \alpha}$, which can be stated as follows.

Theorem 3.7. (Linear exponential stability of wave fronts with critical speeds) Assume either (1.10) holds or (1.7) and $c_{*}^{0}>2 \sqrt{f^{\prime}(0)}$ hold. For each fixed $\alpha^{-} \in\left(0, c_{*}^{0} / 4\right)$ and $\alpha^{+} \in$ $\left(\left(c_{*}^{0}-\sqrt{\left(c_{*}^{0}\right)^{2}-4 f^{\prime}(0)}\right) / 2, c_{*}^{0} / 2\right)$, there exist positive constants $M_{\alpha} \geq 1$, $\delta_{0}$ and $\sigma_{\alpha}$, such that for any $d \in\left[1-\delta_{0}, 1+\delta_{0}\right]$ the analytic semigroup $\mathrm{e}^{t \mathcal{L}_{d, \alpha}^{0}}$ generated by $\mathcal{L}_{d, \alpha}^{0}$ on $Y_{d, \alpha}=$ Range $\left\{\mathcal{L}_{d, c_{*}(d), \alpha}\right\}$ satisfies

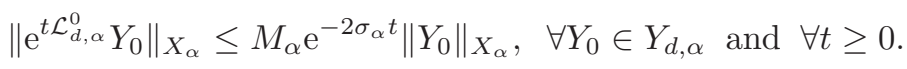


In the following of this section we shall investigate the spectral and linear exponential stability of waves with noncritical speeds $c>c_{*}(d)$ for the case when (1.7) holds and $d$ near 1 . Note that Lemma 3.2 and Lemma 3.5 are still valid for all the wave fronts with noncritical speeds $c>c_{*}(d)$. In the following for the fixed $c>\max \left\{c_{*}(d), c_{*}^{0}\right\}$, we always assume $\alpha^{-} \in(0, c / 2), \alpha^{+} \in\left(\left(c-\sqrt{c^{2}-4 f^{\prime}(0)}\right) / 2, c / 2\right)$, such that Lemma 3.2 holds, further by Theorem 1.2 the slow decaying rate of the wave with a noncritical speed at $z=+\infty$ implies that $\left(W_{c}^{\prime}(z, d), V_{c}^{\prime}(z, d)\right) \notin X_{\alpha}^{2}$; in fact by standard argument (similar proof can be found in [25] or [31] ) it can be proved that zero is not an eigenvalue of $\widetilde{\mathcal{L}}_{1, c, \alpha}$ for any $c>c_{*}^{0}$, and $\widetilde{\mathcal{L}}_{1, c, \alpha}$ has no eigenvalues with nonnegative real parts, which can be stated as follows

Lemma 3.8. Assume (1.7) holds. For any $c>c_{*}^{0}$, let $\alpha^{-} \in(0, c / 4)$ and $\alpha^{+} \in\left(\left(c-\sqrt{c^{2}-4 f^{\prime}(0)}\right) / 2, c / 2\right)$, there exists small positive constant $\delta_{\alpha}$ such that

$$
\sup \left\{\operatorname{Re} \sigma_{p}\left(\widetilde{\mathcal{L}}_{1, c, \alpha}\right)\right\}<-\delta_{\alpha}<0 .
$$

For the fixed $c>\max \left\{c_{*}(d), c_{*}^{0}\right\}$ and $d$ near 1, by (3.16) and (3.19), we can define Evans function $D(\lambda, d, c)$ as in (3.24) by

$$
D(\lambda, d, c)=\exp \left\{-\int_{0}^{z} A(s, \lambda, d, c) \mathrm{d} s\right\} \operatorname{det}\left[Y_{1}^{+} Y_{2}^{+} Y_{3}^{-} Y_{4}^{-}\right](z, \lambda, d, c) ;
$$

which is independent of $z$ and analytic in $\lambda$ for $\lambda \in \Omega_{0}$, with $\Omega_{0}$ defined as before; and $D(\lambda, d, c)=0$ if and only if $\lambda$ is an eigenvalue of $\widetilde{\mathcal{L}}_{d, c, \alpha}$.

By nearly the same argument as in [21] (see the proof of Theorem 1.3 in [21]) it can be proved that $\left(U_{c}(z, d), V_{c}(z, d)\right) \rightarrow\left(U_{c}(z, 1), V_{c}(z, 1)\right)$ in $L_{\infty}$ norm as $d \rightarrow 1$, which implies that $D(\lambda, d, c) \rightarrow D(\lambda, 1, c)$ as $d \rightarrow 1$, then by Lemma 3.8 and by applying Rouchét Theorem it follows that there exists small $\delta_{0}>0$ such that

$$
D(\lambda, d, c) \neq 0, \text { for } \lambda \in \Omega_{0} \text { and }|d-1| \leq \delta_{0},
$$

which guarantees that in $\Omega_{0}$ there exist no eigenvalues of $\widetilde{\mathcal{L}}_{d, c, \alpha}$. Then, by Lemmas 3.2,3.5 and 3.8 and the standard analytic semigroup theories, we have the linear exponential stability of the wave front with speed $c>\max \left\{c_{*}(d), c_{*}^{0}\right\}$ in $X_{\alpha}$ when $d$ is near 1 , more precisely we have

Theorem 3.9. (Linear exponential stability of wave fronts with noncritical speeds for case (1.7)) Assume (1.7) holds. For each fixed $c>c_{*}^{0}$, let $\alpha^{-} \in(0, c / 4)$ and $\alpha^{+} \in\left(\left(c-\sqrt{c^{2}-4 f^{\prime}(0)}\right) / 2, c / 2\right)$, there exist positive constants $M_{\alpha}, \delta_{0}$ and $\sigma_{\alpha}$, such that for any $d \in\left[1-\delta_{0}, 1+\delta_{0}\right]$ the analytic semigroup $\mathrm{e}^{t \mathcal{L}_{d, c, \alpha}}$ generated by $\mathcal{L}_{d, c, \alpha}$ satisfies

$$
\left\|\mathrm{e}^{t \mathcal{L}_{d, c, \alpha}}\right\|_{X_{\alpha} \rightarrow X_{\alpha}} \leq M_{\alpha} \mathrm{e}^{-2 \sigma_{\alpha} t}, \quad \forall t \geq 0 .
$$

\section{Nonlinear stability of waves for $d$ near 1}

Let $\left(U_{c}(x-c t), V_{c}(x-c t)\right)$ be a travelling wave obtained in Theorems 1.1 and 1.2 , which decays exponentially at both ends. In this section, we investigate the nonlinear asymptotic stability of the wave $\left(U_{c}(x-c t), V_{c}(x-c t)\right)$ in some weighted and unweighted spaces when $d$ is near 1.

In moving coordinate $z=x-c t$, let $(u(z, t), v(z, t))$ be the solution of the nonlinear system (3.1) with initial data $\left(u_{0}(z), v_{0}(z)\right)$, and define $\hat{u}(z, t)=u(z, t)-U_{c}(z)$ and $\hat{v}(z, t)=v(z, t)-V_{c}(z)$, then $(\hat{u}(z, t), \hat{v}(z, t))$ satisfies

$$
\left\{\begin{array}{l}
\left(\begin{array}{l}
\hat{u}_{t}(z, t) \\
\hat{v}_{t}(z, t)
\end{array}\right)=\mathcal{L}_{d, c}\left(\begin{array}{l}
\hat{u}(z, t) \\
\hat{v}(z, t)
\end{array}\right)+\left(\begin{array}{l}
-f_{1}(\hat{u}, \hat{v}) \\
f_{1}(\hat{u}, \hat{v})
\end{array}\right), \quad z \in \mathbb{R}, t>0, \\
(\hat{u}(z, 0), \hat{v}(z, 0))=\left(u_{0}(z)-U_{c}(z), v_{0}(z)-V_{c}(z)\right), \quad z \in \mathbb{R} ;
\end{array}\right.
$$


with $\mathcal{L}_{d, c}$ defined by $(3.2)$, and

$$
\left\{\begin{array}{l}
f_{1}(\hat{u}, \hat{v})=U_{c} h_{1}\left(V_{c}, \hat{v}\right)+\hat{u} h_{2}\left(V_{c}, \hat{v}\right) \\
h_{1}\left(V_{c}, \hat{v}\right)=f\left(V_{c}+\hat{v}\right)-f\left(V_{c}\right)-f^{\prime}\left(V_{c}\right) \hat{v} \text { and } h_{2}\left(V_{c}, \hat{v}\right)=f\left(V_{c}+\hat{v}\right)-f\left(V_{c}\right)
\end{array}\right.
$$

satisfying

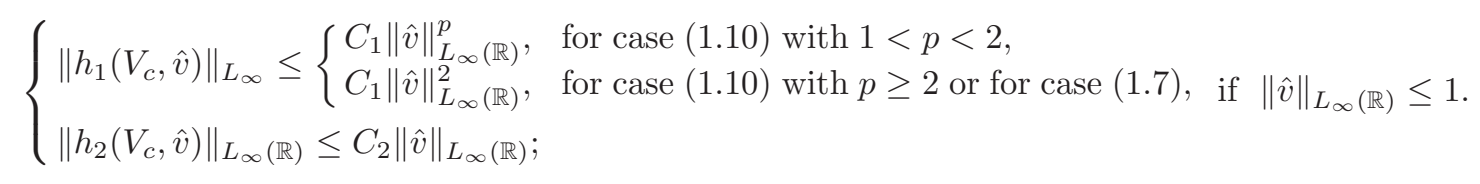

Let $X=C_{\text {unif }}(\mathbb{R}) \times C_{\text {unif }}(\mathbb{R})$, and for $\alpha^{-}>0$ and $\alpha^{+}>0$ define weight spaces $C_{\alpha}(\mathbb{R}), \mathcal{B}_{\alpha^{+}}(\mathbb{R}), X_{\alpha}$ and $X_{\alpha}^{2}$ as in Section 1 and Section 3.

We shall first investigate the evolution of the perturbation of the wave with the critical speed $c=c_{*}(d)$ in $X$ and $X_{\alpha}$ when the initial perturbation of the wave is small in the weighted space $\mathcal{B}_{\alpha^{+}}(\mathbb{R}) \times \mathcal{B}_{\alpha^{+}}(\mathbb{R})=$ $X \cap X_{\alpha}$, with the pair $\alpha=\left(\alpha^{-}, \alpha^{+}\right)$satisfying the assumptions in Theorem 3.7.

For the fixed $d$ near 1 , introducing new variables $\gamma(t) \in \mathbb{R}$ and $Y(z, t)=\left(y_{1}(z, t), y_{2}(z, t)\right)^{T} \in Y_{d, \alpha}=$ Range $\left(L_{d, c, \alpha}\right)$ such that

$$
\begin{gathered}
Y(z, t)=(u(z, t), v(z, t))^{T}-\Phi_{c}(z+\gamma(t))=(\hat{u}(z, t), \hat{v}(z, t))^{T}-\hat{\Phi}_{c}(z, \gamma(t)) \text { for } t>0 \\
Y(z, 0)=\left(u_{0}(z)-U_{c}\left(z+\gamma_{0}\right), v_{0}(z)-V_{c}\left(z+\gamma_{0}\right)\right)^{T} \triangleq Y_{0} \in Y_{d, \alpha}, \text { and } \gamma(0)=\gamma_{0}
\end{gathered}
$$

with $\Phi_{c}(z)=\left(U_{c}(z, d), V_{c}(z, d)\right)^{T}, c=c_{*}(d)$ and

$$
\hat{\Phi}_{c}(z, \gamma(t))=\left(U_{c}(z+\gamma(t))-U_{c}(z), V_{c}(z+\gamma(t))-V_{c}(z)\right)^{T} \triangleq\left(\hat{U}_{c}(z, \gamma(t)), \hat{V}_{c}(z, \gamma(t))\right)^{T} .
$$

Then system (4.1) becomes

$$
\begin{cases}Y_{t}+\gamma^{\prime}(t) \Phi_{c}^{\prime}(z+\gamma(t))=\mathcal{L}_{d, \alpha}^{0} Y+H(z, t, Y, \gamma), & z \in \mathbb{R}, t>0 \\ Y(z, 0)=Y_{0}(z), \gamma(0)=\gamma_{0}, & z \in \mathbb{R}\end{cases}
$$

where $H(z, t, Y, \gamma)=\left(-H_{1}(z, t, Y, \gamma), H_{1}(z, t, Y, \gamma)\right)^{T}$ and

$$
\begin{aligned}
& H_{1}(z, t, Y, \gamma)=U_{c}(z+\gamma(t)) h_{1}\left(V_{c}(z, \gamma(t)), y_{2}(z, t)\right)+y_{1}(z, t)\left[h_{2}\left(V_{c}(z, \gamma(t)), y_{2}(z, t)\right)\right. \\
& \quad+y_{1}(z, t)\left(f\left(V_{c}(z, \gamma(t))-f\left(V_{c}(z)\right)\right)+y_{2}\left[U_{c}(z, \gamma(t)) f^{\prime}\left(V_{c}(z, \gamma(t))\right)-U_{c}(z) f^{\prime}\left(V_{c}(z)\right)\right],\right.
\end{aligned}
$$

with $h_{1}$ and $h_{2}$ defined by (4.2).

By (1.11), (4.3) and (4.7), it can be verified that

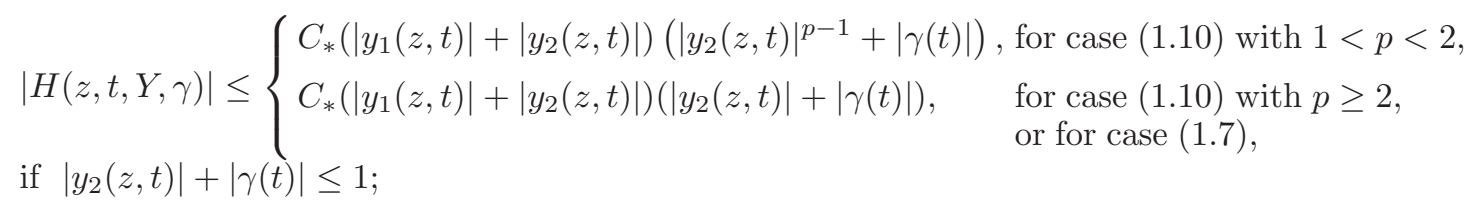

with some positive constant $C_{*}$ independent of $Y, \gamma, z$ and $t$.

(4.8) also implies that there exists a positive constant $C$ such that

$$
\|H(\cdot, t, Y, \gamma)\|_{X} \leq\left\{\begin{array}{lr}
C \varepsilon_{0}^{p-1}\|Y(\cdot, t)\|_{X}, & \text { for case }(1.10) \text { with } 1<p<2 \\
C \varepsilon_{0}\|Y(\cdot, t)\|_{X}, & \text { for case }(1.10) \text { with } p \geq 2, \text { or for case }(1.7)
\end{array}\right.
$$


and for any $\alpha^{ \pm} \geq 0$ it holds that

$$
\begin{aligned}
& \|H(\cdot, t, Y, \gamma)\|_{X_{\alpha}} \leq\left\{\begin{array}{l}
C \varepsilon_{0}^{p-1}\|Y(\cdot, t)\|_{X_{\alpha}}, \text { for case }(1.10) \text { with } 1<p<2, \\
C \varepsilon_{0}\|Y(\cdot, t)\|_{X_{\alpha}}, \quad \text { for case }(1.10) \text { with } p \geq 2, \text { or for case }(1.7) ;
\end{array}\right. \\
& \text { if }\left\|y_{2}(\cdot, t)\right\|_{L_{\infty}(\mathbb{R})}+|\gamma(t)| \leq \varepsilon_{0} \leq 1 .
\end{aligned}
$$

(4.6) with the selection of $Y(t) \in Y_{d, \alpha}$ implies that $Y(t)$ and $\gamma(t)$ satisfy the following initial value problems

$$
\left\{\begin{array}{l}
\gamma^{\prime}(t)=\frac{\rho(H(Y(z, t), \gamma(t)))}{\rho\left(\Phi_{c}^{\prime}(z+\gamma(t))\right)} \triangleq \kappa(Y(\cdot, t), \gamma(t)), \quad t>0 \\
\gamma(0)=\gamma_{0}
\end{array}\right.
$$

and

$$
\begin{cases}Y_{t}=\mathcal{L}_{d, \alpha}^{0} Y+H_{2}(z, t, Y, \gamma), & z \in \mathbb{R}, t>0, \\ Y(z, 0)=Y_{0}(z), & z \in \mathbb{R}\end{cases}
$$

with $\rho$ defined by (3.26), $\gamma_{0}$ defined by (4.5) and

$$
H_{2}(z, t, Y, \gamma)=H(z, t, Y, \gamma)-\kappa(Y(\cdot, t), \gamma(t)) \Phi_{c}^{\prime}(z+\gamma(t)) \in Y_{d, \alpha} .
$$

Before proving Theorem 1.4, by virtue of Theorem 3.7 we shall first prove that under the assumption of the smallness of the perturbation $(Y(t), \gamma(t))$ in $X \times R$ norm, the solution $(Y(t), \gamma(t))$ to nonlinear system (4.11)-(4.12) satisfies some exponential decay estimates in $X_{\alpha} \times R$ norm.

Proposition 4.1. Assume either (1.10) holds or (1.7) and $c_{*}^{0}>4 \sqrt{f^{\prime}(0)}$ hold, for small enough $\alpha^{-}>0$ and $\alpha^{+}>0$ satisfying the assumptions of Theorem 3.7, let $\varepsilon_{0} \in(0,1)$ be small enough and satisfies

$$
\begin{cases}\varepsilon_{0} \leq \frac{\sigma_{\alpha}}{4 C C_{0}^{2} M_{\alpha}}, & \text { for case (1.10) with } p \geq 2 \text {, or for case (1.7), } \\ \text { or } \varepsilon_{0}^{p-1} \leq \frac{\sigma_{\alpha}}{4 C C_{0}^{2} M_{\alpha}}, & \text { for case (1.10) with } 1<p<2 ;\end{cases}
$$

with positive constants $C_{0}, C, \sigma_{\alpha}$ and $M_{\alpha}$ defined as in (4.18), (3.29) and (4.10). For any initial data $Y_{0} \in X \cap Y_{\alpha, d}$ and $\gamma_{0}$ satisfying $\left\|Y_{0}\right\|_{X}+\left|\gamma_{0}\right|<\varepsilon_{0}$, let $(Y(z, t), \gamma(t)) \in C\left(\left[0, T_{\max }\right),\left(X \cap Y_{\alpha, d}\right) \times R\right)$ denote the unique maximal solution to the initial value problem (4.11)-(4.12), if for some $0<T \leq T_{\max }$ it holds that

$$
\|Y(z, t)\|_{X}+|\gamma(t)| \leq \varepsilon_{0}, \quad \forall t \in[0, T)
$$

then

$$
\|Y(z, t)\|_{X_{\alpha}}+\left|\gamma^{\prime}(t)\right| \leq 2 C_{\alpha}^{0} \mathrm{e}^{-\sigma_{\alpha} t}\left\|Y_{0}\right\|_{X_{\alpha}}, \quad \forall t \in[0, T),
$$

and

$$
|\gamma(t)| \leq\left|\gamma_{0}\right|+2 \frac{C_{\alpha}^{0}}{\sigma_{\alpha}}\left\|Y_{0}\right\|_{X_{\alpha}}, \forall t \in[0, T)
$$

with $C_{\alpha}^{0}$ some positive constant independent of $Y_{0}, \varepsilon_{0}, \gamma_{0}, t$ and $T$.

Proof. For any initial data $Y_{0} \in X \cap Y_{\alpha, d}$ and small $\left|\gamma_{0}\right|$, the existence and uniqueness of a maximal solution $(Y(z, t), \gamma(t)) \in C\left(\left[0, T_{\max }\right),\left(X \cap Y_{d, \alpha}\right) \times R\right)$ follow from the standard analytic semigroup theories, and the smallness of $\left\|Y_{0}\right\|_{X}+\left|\gamma_{0}\right|$ also implies that the estimate (4.15) holds true for some $T>0$.

Note that $w_{\alpha}(z) \Phi_{0}^{\prime}(z) \rightarrow 0$ exponentially as $z \rightarrow \pm \infty$ for small $\alpha^{-}>0$, and $\rho\left(\Phi_{c}^{\prime}(z)\right)=1$. If the assumption of (4.15) holds for small enough $\varepsilon_{0}>0$, then it is easy to prove that

$$
\frac{1}{2} \leq \rho\left(\Phi_{c}^{\prime}(z+\gamma(t)) \leq \frac{3}{2}, \quad \forall t \in[0, T)\right.
$$

and there exists a constant $C_{0}$ such that

$$
|\rho(Y)| \leq C_{0}\|Y\|_{X_{\alpha}} \quad \forall Y \in X_{\alpha} ; \text { and } C_{0}^{-1} \leq \frac{\rho\left(\Phi_{c}^{\prime}(z+\sigma(t))\right)}{\left\|\Phi_{c}^{\prime}(z+\sigma(t))\right\|_{X_{\alpha}}} \leq C_{0}, \quad \forall t \in[0, T) ;
$$


which with (4.10) further implies

$$
|\kappa(H(z, t, Y, \gamma))| \leq\left\{\begin{array}{l}
2 C C_{0} \varepsilon_{0}^{p-1}\|Y(z, t)\|_{X_{\alpha}}, \text { for case (1.10) with } 1<p<2, \\
2 C C_{0} \varepsilon_{0}\|Y(z, t)\|_{X_{\alpha}}, \quad \text { for case (1.10) with } p \geq 2, \text { or for case (1.7); }
\end{array} \quad \forall t \in[0, T) ;\right.
$$

and

$$
\left\|H_{2}(z, t, Y, \gamma)\right\|_{X_{\alpha}} \leq\left\{\begin{array}{l}
2 C C_{0}^{2} \epsilon_{0}^{p-1}\|Y(z, t)\|_{X_{\alpha}}, \text { for case (1.10) with } 1<p<2, \\
2 C C_{0}^{2} \epsilon_{0}\|Y(z, t)\|_{X_{\alpha}}, \quad \text { for case (1.10) with } p \geq 2, \text { or for case (1.7); }
\end{array} \quad \forall t \in[0, T) .\right.
$$

By Theorem 3.7, for any $t \in\left[0, T_{\max }\right)$ the solution $(Y(t), \gamma(t)) \in Y_{d, \alpha} \times R$ to system (4.11)-(4.12) satisfies

$$
\begin{gathered}
\|Y(t)\|_{X_{\alpha}} \leq\left\|\mathrm{e}^{t \mathcal{L}_{d, \alpha}^{0}} Y_{0}\right\|_{X_{\alpha}}+\int_{0}^{t}\left\|\mathrm{e}^{(t-s) \mathcal{L}_{d, \alpha}^{0}} Q_{\alpha}^{d} H_{2}(\cdot, s, Y, \gamma)\right\|_{X_{\alpha}} \mathrm{d} s \\
\leq M_{\alpha} \mathrm{e}^{-2 \sigma_{\alpha} t}\left\|Y_{0}\right\|_{X_{\alpha}}+M_{\alpha} \int_{0}^{t} \mathrm{e}^{-2 \sigma_{\alpha}(t-s)}\left\|H_{2}(\cdot, s, Y, \gamma)\right\|_{X_{\alpha}} \mathrm{d} s .
\end{gathered}
$$

For any $t \in[0, T)$ let $K_{1}(t)=\sup _{0 \leq s \leq t} \mathrm{e}^{\sigma_{\alpha} s}\|Y(z, s)\|_{X_{\alpha}}$, then by (4.14), (4.15),(4.20) and (4.21), we have

$$
K_{1}(t) \leq M_{\alpha}\left\|Y_{0}\right\|_{X_{\alpha}}+\frac{\sigma_{\alpha}}{2} K_{1}(t) \int_{0}^{t} \mathrm{e}^{-\sigma_{\alpha}(t-s)} \mathrm{d} s, \quad t \in[0, T)
$$

thus

$$
K_{1}(t) \leq 2 M_{\alpha}\left\|Y_{0}\right\|_{X_{\alpha}}, \quad \forall t \in[0, T),
$$

which with (4.11) and (4.19) implies estimates (4.16) and (4.17) hold. This completes the proof of Proposition 4.1.

The main idea of the proof of Theorem 1.4 is similar to that in [12], in which the similar nonlinear stability results were obtained for more general R-D systems under a series of abstract assumptions. It is worth mentioning that the detailed proof in [12] about the decaying estimates of the perturbation of waves in the unweighted spaces is rather long and not standard, which is also closely related with the special form of the system, some additional spectral estimates and some abstract assumptions. Instead of verifying all the abstract assumptions in [12], in the following we shall combine the main idea of the proof in [12] with some additional estimates for our system to give a complete proof of the nonlinear stability of the waves with critical speeds when $d$ is near 1 .

Proof of Theorem 1.4. (i) For the fixed $d$ near 1, to prove the exponential decay of the perturbation of the waves with the critical speed $c=c_{*}(d)$ in $X_{\alpha}$ for small $\alpha_{-}>0$ and fixed $\alpha^{+} \in\left(\alpha_{-}^{0}, \alpha_{+}^{0}\right)$, it suffices to prove that for each fixed small $\varepsilon_{0}>0$ satisfying (4.14), there exists a small positive constant $\delta_{\alpha}^{0}$ such that if the initial perturbation satisfies

$$
\left\|\left(u_{0}(z)-U_{c_{*}(d)}(z, d), v_{0}(z)-V_{c_{*}(d)}(z, d)\right)\right\|_{\mathcal{B}_{\alpha+}} \leq \delta_{\alpha}^{0},
$$

then there exists a unique global solution $(Y(z, t), \gamma(t)) \in C\left([0, \infty),\left(X \cap Y_{d, \alpha}\right) \times R\right)$ to system (4.11)(4.12), and estimate (4.15) holds for all $t \geq 0$; which with Proposition 4.1 will further assures that (4.16) holds for all $t \geq 0$. Estimate (4.15) for all $t \geq 0$ can be proved by combing the main idea of proof in [12] with the similar estimates in [21]. Here we just give the outline of the proof.

By nearly the same argument as in [12] (see the detailed proof in Sect.5.4 [12]), it follows that for each fixed small $\delta_{*}>0$ there exists small enough $\delta_{\alpha}^{0}>0$, such that for any $\left(u_{0}, v_{0}\right)$ satisfying (4.22) there exists a unique $\left(Y_{0}(z), \gamma_{0}\right) \in\left(Y_{d, \alpha} \cap X\right) \times R$ satisfying

$$
\left(u_{0}(z), v_{0}(z)\right)=\left(U_{c_{*}(d)}\left(z+\gamma_{0}, d\right), V_{c_{*}(d)}\left(z+\gamma_{0}, d\right)\right)+Y_{0}(z), z \in \mathbb{R},
$$


and

$$
\left\|Y_{0}\right\|_{X}+\left\|Y_{0}\right\|_{X_{\alpha}}+\left|\gamma_{0}\right| \leq \delta_{*}<\varepsilon_{0} .
$$

Let $(Y(z, t), \gamma(t))$ be the unique solution of system (4.11)-(4.12) defined on the maximal existence interval $\left(0, T_{\max }\right)$ with the initial data $\left(Y_{0}, \gamma_{0}\right)$, which satisfies $(4.15)$ on $[0, T)$ for some $T \in\left(0, T_{\max }\right]$. Denote $\widehat{y}_{1}(z, t)=w_{\alpha}(z) y_{1}(z, t)$ and $\widehat{y}_{2}(z, t)=w_{\alpha}(z) y_{2}(z, t)$, we can rewrite the equation of $y_{1}(z, t)$ in system (4.12) as

$$
\begin{aligned}
y_{1 t}(z, t)= & d y_{1 z z}(z, t)+c y_{1 z}(z, t)-y_{1}(z, t)+y_{1}(z, t)\left[f(1)-f\left(V_{c}(z+\gamma)+y_{2}(z, t)\right)\right] \\
& -U_{c}(z+\gamma)\left[f\left(V_{c}(z+\gamma)\right)-f\left(V_{c}(z+\gamma)+y_{2}\right)\right]-U_{c}^{\prime}(z+\gamma) \gamma^{\prime}(t) \\
= & L_{1} y_{1}+\widehat{y}_{1}(z, t) w_{\alpha}^{-1}(z)\left[1-f\left(V_{c}(z+\gamma)\right)\right]-y_{1}(z, t) y_{2}(z, t)\left[f^{\prime}\left(V_{c}(z+\gamma)\right)+h_{1}\left(V_{c}(z+\gamma), y_{2}\right)\right] \\
& -\left[w_{\alpha}^{-1}(z) U_{c}(z+\gamma) \widehat{y}_{2}(z, t)\left[f^{\prime}\left(V_{c}(z, \gamma(t))\right)+h_{1}\left(V_{c}(z+\gamma), y_{2}(z, t)\right)\right]-U_{c}^{\prime}(z, \gamma(t)) \gamma^{\prime}(t)\right. \\
= & L_{1} y_{1}+H_{1}(z, t, Y, \gamma(t))+g(z, t, \gamma)
\end{aligned}
$$

where the operator $L_{1}$ is defined by $L_{1}=d \frac{\partial^{2}}{\partial z^{2}}+c \frac{\partial}{\partial z}-1, g(z, t, \gamma)=-U_{c}^{\prime}(z, \gamma(t)) \gamma^{\prime}(t)$ and

$$
H_{1}(z, t, Y, \gamma)=g_{1}(z, \gamma) \widehat{y}_{1}(z, t)+g_{2}\left(y_{2}, z, \gamma\right) y_{1}(z, t) y_{2}(z, t)+g_{3}\left(y_{2}, z, \gamma\right) \widehat{y}_{2}(z, t),
$$

with

$$
\begin{gathered}
g_{1}(z, \gamma)=w_{\alpha}^{-1}(z)\left(f(1)-f\left(V_{c}(z, \gamma(t))\right)\right), g_{2}\left(y_{2}, z, \gamma\right)=-f^{\prime}\left(V_{c}(z, \gamma(t))\right)-h_{1}\left(y_{2}, z+\gamma\right), \\
\text { and } g_{3}\left(y_{2}, z, \gamma\right)=-w_{\alpha}^{-1}(z) U_{c}(z, \gamma(t))\left[f^{\prime}\left(V_{c}(z, \gamma(t))\right)+h_{1}\left(V_{c}(z+\gamma), y_{2}(z+\gamma)\right) .\right.
\end{gathered}
$$

Note that $\operatorname{Re} \sigma\left(L_{1}\right) \leq-1$, which implies that for each fixed $b \in(0,1)$, there exists constant $C_{b}$ such that

$$
\left\|\mathrm{e}^{L_{1} t}\right\|_{C_{\text {unif }}(\mathbb{R}) \rightarrow C_{\text {unif }}(\mathbb{R})} \leq C_{b} \mathrm{e}^{-b t}, \quad\left\|\mathrm{e}^{L_{1} t}\right\|_{L_{1}(\mathbb{R}) \rightarrow L_{1}(\mathbb{R})} \leq C_{b} \mathrm{e}^{-b t}, \quad \forall t \geq 0 .
$$

It follows from Theorem 1.1 that

$$
\begin{aligned}
& w_{\alpha}^{-1}(z) U_{c}(z, d) \rightarrow 0, \quad w_{\alpha}^{-1}(z)\left[V_{c}(z, d)-1\right] \rightarrow 0, \text { exponentially as } z \rightarrow \pm \infty, \\
& \text { for small enough } \alpha^{-}>0 \text { and } \alpha^{+} \in\left(\alpha_{-}^{0}, \alpha_{0}^{+}\right)
\end{aligned}
$$

which with the assumption (4.15) for small $\varepsilon_{0}>0$, further implies that there exist positive constants $K_{0}$ and $K_{1}$ independent of $d, T$ and $\varepsilon_{0}$ such that

$$
\left\{\begin{array}{l}
\left\|g_{1}(z, \gamma)\right\|_{L_{\infty}(\mathbb{R})}+\left\|g_{3}\left(y_{2}, z, \gamma\right)\right\|_{L_{\infty}(\mathbb{R})} \leq K_{0}, \\
\left\|g_{1}(z, \gamma)\right\|_{L_{1}(\mathbb{R})}+\left\|g_{3}\left(y_{2}, z, \gamma\right)\right\|_{L_{1}(\mathbb{R})} \leq K_{0}, \quad \forall t \in[0, T) \\
\left\|g_{2}\left(y_{2}, z, \gamma\right)\right\|_{L_{\infty}(\mathbb{R})} \leq K_{1},
\end{array}\right.
$$

thus we have

$$
\left\{\begin{array}{l}
\left\|H_{1}(\cdot, t, Y, \gamma)\right\|_{L_{\infty}(\mathbb{R})} \leq K_{0}\|Y(\cdot, t)\|_{X_{\alpha}}+K_{1} \varepsilon_{0}\left\|y_{1}(\cdot, t)\right\|_{L_{\infty}(\mathbb{R})}, \\
\left\|H_{1}(\cdot, t, Y, \gamma)\right\|_{L_{1}} \leq K_{0}\|Y(\cdot, t)\|_{X_{\alpha}}+K_{1} \varepsilon_{0}\left\|y_{1}(\cdot, t)\right\|_{L_{1}},
\end{array} \forall t \in[0, T) .\right.
$$

Further, by Proposition 4.1, we have

$$
\|Y(\cdot, t)\|_{X_{\alpha}},\|g(z, t, \gamma)\|_{L_{\infty}(\mathbb{R})},\|g(z, t, \gamma)\|_{L_{1}(\mathbb{R})} \leq K_{2} \mathrm{e}^{-\sigma_{\alpha} t}\left\|Y_{0}\right\|_{X_{\alpha}}, \forall t \in[0, T) ;
$$

for some constant $K_{2}$ independent $d, Y_{0}, T$ and $\varepsilon_{0}$. 
Rewrite equation (4.24) as

$$
y_{1}(\cdot, t)=\mathrm{e}^{L_{1} t} y_{1}^{0}(\cdot)+\int_{0}^{t} \mathrm{e}^{L_{1}(t-s)}\left[H_{1}(\cdot, s, Y, \gamma)+g(\cdot, s, \gamma)\right] \mathrm{d} s,
$$

then by (4.25)-(4.29), under assumption (4.15) we have

$$
\begin{aligned}
\left\|y_{1}(\cdot, t)\right\|_{L_{\infty}(\mathbb{R})} \leq & C_{b} \mathrm{e}^{-b t}\left\|y_{1}^{0}\right\|_{L_{\infty}(\mathbb{R})}+C_{b} \int_{0}^{t} \mathrm{e}^{-b(t-s)}\left(\left\|H_{1}(\cdot, s, Y, \gamma)\right\|_{L_{\infty}(\mathbb{R})}+\|g(\cdot, s, \gamma)\|_{L_{\infty}(\mathbb{R})}\right) \mathrm{d} s \\
\leq & C_{b} \mathrm{e}^{-b t}\left\|y_{1}^{0}\right\|_{L_{\infty}(\mathbb{R})}+C_{b} K_{1} \varepsilon_{0} \int_{0}^{t} \mathrm{e}^{-b(t-s)}\left\|y_{1}(\cdot, s)\right\|_{L_{\infty}(\mathbb{R})} \mathrm{d} s \\
& +C_{b}\left(K_{0}+1\right) K_{2}\left\|Y_{0}\right\|_{X_{\alpha}} \int_{0}^{t} \mathrm{e}^{-b(t-s)} \mathrm{e}^{-\sigma_{\alpha} s} \mathrm{~d} s ; \quad \forall t \in[0, T) .
\end{aligned}
$$

By choosing $\sigma=\frac{\sigma_{\alpha}}{2}>0$ small enough such that $2 \sigma<b<1$, then by (4.30) it follows that

$$
\begin{aligned}
\mathrm{e}^{\sigma t}\left\|y_{1}(\cdot, t)\right\|_{L_{\infty}(\mathbb{R}) \leq} & C_{b} \mathrm{e}^{-(b-\sigma) t}\left\|y_{1}^{0}\right\|_{L_{\infty}(\mathbb{R})}+C_{b}\left(K_{0}+1\right) K_{2}\left\|Y_{0}\right\|_{X_{\alpha}} \int_{0}^{t} \mathrm{e}^{-b(t-s)} \mathrm{e}^{\sigma t-2 \sigma s} \mathrm{~d} s \\
& +C_{b} \varepsilon_{0} K_{1} \int_{0}^{t} \mathrm{e}^{-b(t-s)} \mathrm{e}^{\sigma t}\left\|y_{1}(\cdot, s)\right\|_{L_{\infty}(\mathbb{R})} \mathrm{d} s \\
\leq & C_{b} \mathrm{e}^{-\sigma t}\left\|y_{1}^{0}\right\|_{L_{\infty}(\mathbb{R})}+\frac{C_{b}\left(K_{0}+1\right) K_{2}}{b-2 \sigma}\left\|Y_{0}\right\|_{X_{\alpha}} \\
& +C_{b} \varepsilon_{0} K_{1} \int_{0}^{t} \mathrm{e}^{-(b-\sigma)(t-s)}\left\|\mathrm{e}^{\sigma s} y_{1}(\cdot, s)\right\|_{L_{\infty}(\mathbb{R})} \mathrm{d} s, \quad \forall t \in[0, T) .
\end{aligned}
$$

Let $K(t)=\sup _{0 \leq s \leq t} \mathrm{e}^{\sigma s}\|u(\cdot, s)\|_{L_{\infty}(\mathbb{R})}$, then

$$
K(t) \leq C_{b}\left\|y_{1}^{0}\right\|_{L_{\infty}(\mathbb{R})}+\frac{C_{b}\left(K_{0}+1\right) K_{2}}{b-2 \sigma}\left\|Y_{0}\right\|_{X_{\alpha}}+\frac{C_{b} \varepsilon_{0} K_{1}}{b-\sigma} K(t), \quad \forall t \in[0, T) .
$$

Further, by choosing $\varepsilon_{0}>0$ small enough such that

$$
\varepsilon_{0} \leq \frac{b-\sigma}{2 C_{b} K_{1}}
$$

then we have

$$
K(t) \leq 2 C_{b}\left\|y_{1}^{0}\right\|_{L_{\infty}(\mathbb{R})}+2 \frac{C_{b}\left(K_{0}+1\right) K_{2}}{b-2 \sigma}\left\|Y_{0}\right\|_{X_{\alpha}}, \quad \forall t \in[0, T),
$$

which proves that under the assumption that

$$
\|Y(\cdot, t)\|_{X}+|\gamma(t)| \leq \varepsilon_{0}, \quad \forall t \in[0, T), \text { with } \varepsilon_{0} \text { satisfying (4.15) and (4.31); }
$$

it holds that

$$
\left\|y_{1}(\cdot, t)\right\|_{L_{\infty}(\mathbb{R})} \leq C_{2} \mathrm{e}^{-\frac{\sigma_{\alpha}}{2} t}\left(\left\|y_{1}^{0}\right\|_{L_{\infty}(\mathbb{R})}+\left\|Y_{0}\right\|_{X_{\alpha}}\right), \quad \forall t \in[0, T),
$$

with $C_{2}$ depending only on $\alpha$ and $\sigma_{\alpha}$.

Now rewrite the $y_{2}$ equation in (4.12) as

$$
y_{2 t}=L_{2} y_{2}+\tilde{H}_{2}(z, t, Y, \gamma)+G_{2}(z, t, \gamma)
$$

with $L_{2}=\frac{\partial^{2}}{\partial z^{2}}+c \frac{\partial}{\partial z}, G_{2}(z, t, \gamma)=V_{c}^{\prime}(z, \gamma(t)) \gamma^{\prime}(t)$ and

$$
\tilde{H}_{2}(z, t, Y, \gamma)=y_{1} f\left(V_{c}(z+\gamma)+y_{2}\right)+\widehat{y}_{2} w_{\alpha}^{-1}(z) U_{c}(z+\gamma)\left[f^{\prime}\left(V_{c}(z+\gamma)\right)+\frac{h_{1}\left(V_{c}(z+\gamma), y_{2}(z, t)\right)}{y_{2}(z, t)}\right]
$$


where $h_{1}$ is defined as in (4.2).

By (1.10), (1.7) and (4.3), under the assumption of (4.32) we have

$$
\left\{\begin{array}{l}
\left\|\tilde{H}_{2}(\cdot, t, Y, \gamma)\right\|_{L_{\infty}(\mathbb{R})}+\left\|G_{2}(z, t, \gamma)\right\|_{L_{\infty}(\mathbb{R})} \leq C_{2}^{0}\left(\left\|y_{1}(\cdot, t)\right\|_{L_{\infty}(\mathbb{R})}+\left\|\widehat{y}_{2}(\cdot, t)\right\|_{L_{\infty}(\mathbb{R})}+\left|\gamma^{\prime}(t)\right|\right), \\
\left\|\tilde{H}_{2}(\cdot, t, Y, \gamma)\right\|_{L_{1}(\mathbb{R})}+\left\|G_{2}(z, t, \gamma)\right\|_{L_{1}(\mathbb{R})} \leq C_{2}^{0}\left(\left\|y_{1}(\cdot, t)\right\|_{L_{1}(\mathbb{R})}+\left\|\widehat{y}_{2}(\cdot, t)\right\|_{L_{\infty}(\mathbb{R})}+\left|\gamma^{\prime}(t)\right|\right),
\end{array} \quad \forall t \in[0, T) ;\right.
$$

with constant $C_{2}^{0}$ independent of $\epsilon_{0}$ and $T$. (4.16), (4.33) and (4.35) further imply that there exists a constant $K$ independent of $Y_{0}$ and $T$ such that

$$
\left\|\tilde{H}_{2}(\cdot, t, Y, \gamma)\right\|_{L_{\infty}(\mathbb{R})}+\left\|G_{2}(z, t, \gamma)\right\|_{L_{\infty}(\mathbb{R})} \leq K \mathrm{e}^{-\frac{\sigma_{\alpha}}{2} t}\left(\left\|y_{1}^{0}\right\|_{L_{\infty}(\mathbb{R})}+\left\|Y_{0}\right\|_{X_{\alpha}}\right), \quad \forall t \in[0, T) ;
$$

then using the fact that $\left\|\mathrm{e}^{L_{2} t}\right\|_{L_{\infty} \rightarrow L_{\infty}} \leq 1$ for any $t \geq 0$, it follows from (4.34) that

$$
\begin{aligned}
\left\|y_{2}(\cdot, t)\right\|_{L_{\infty}} & \leq\left\|\mathrm{e}^{L_{2} t} y_{2}^{0}\right\|_{L_{\infty}}+K \int_{0}^{t}\left\|\mathrm{e}^{L_{2}(t-s)}\left(\tilde{H}_{2}(\cdot, s, Y(s), \gamma(s))+G_{2}(z, s, \gamma(s))\right)\right\|_{L_{\infty}(\mathbb{R})} \mathrm{d} s \\
& \leq\left\|y_{2}^{0}\right\|_{L_{\infty}}+K \int_{0}^{t} \mathrm{e}^{-\sigma_{\alpha} s / 2} \mathrm{~d} s\left(\left\|y_{1}^{0}\right\|_{L_{\infty}(\mathbb{R})}+\left\|Y_{0}\right\|_{X_{\alpha}}\right) \\
& \leq \frac{2 K}{\sigma_{\alpha}}\left(\left\|Y_{0}\right\|_{X}+\left\|Y_{0}\right\|_{X_{\alpha}}\right), \quad \forall t \in[0, T) ;
\end{aligned}
$$

which with (4.33) and (4.17) further implies that

$$
\|Y(\cdot, t)\|_{X}+|\gamma(t)| \leq M\left(\left\|Y_{0}\right\|_{X}+\left\|Y_{0}\right\|_{X_{\alpha}}+\left|\gamma_{0}\right|\right), \quad \forall t \in[0, T),
$$

with constant $M$ independent of $\varepsilon_{0}, T, Y_{0}$ and $\gamma_{0}$.

For the fixed $\varepsilon_{0}>0$ satisfying (4.14) and (4.31), then (4.38) implies that if initial value $\left(Y_{0}, \gamma_{0}\right)$ satisfies

$$
\left\|Y_{0}\right\|_{X}+\left\|Y_{0}\right\|_{X_{\alpha}}+\left|\gamma_{0}\right| \leq \delta_{\alpha}^{0}=\frac{\varepsilon_{0}}{2 M}
$$

and if $\|Y(z, t)\|_{X}+|\gamma(t)| \leq \varepsilon_{0}$ holds on $[0, T)$ for some maximal $T \in\left(0, T_{\max }\right]$, then

$$
\|Y(z, t)\|_{X}+|\gamma(t)| \leq \frac{\varepsilon_{0}}{2}, \quad \forall t \in[0, T),
$$

which further implies for any initial value $\left(Y_{0}, \gamma_{0}\right)$ small enough satisfying (4.39), then the solution $(Y(z, t), \gamma(t))$ to system (4.11)-(4.12) exists globally and estimate (4.15) holds for all $t \geq 0$, thus (4.16),(4.17) (4.33) and (4.38) are true for all $t \geq 0$.

Note that (4.16) with $T=\infty$ also guarantees that there exists $\gamma_{*} \in \mathbb{R}$ such that

$$
\mathrm{e}^{\frac{\sigma_{\alpha}}{2}}\left|\gamma(t)-\gamma_{*}\right| \rightarrow 0 \text { as } t \rightarrow \infty
$$

thus (1.18) holds, and (1.17) follows from (4.23), (4.33) and (4.38). This completes the proof of Theorem 1.4 (i).

(ii) Under the assumption of the smallness of the initial perturbation of the wave in $X \cap X_{\alpha}$, in (i) we have shown that there exists a unique global in time classical solution $(Y(z, t), \gamma(t))$ to system (4.11)(4.12) satisfying (4.16),(4.33) and (4.38) for any $t \geq 0$. In the following we shall further assume that the initial perturbation is in addition small in $L_{1}(\mathbb{R}) \times L_{1}(\mathbb{R})$ space, i.e. for small enough $\delta_{\alpha}^{0}>0$ the initial data $\left(Y_{0}, \gamma_{0}\right)$ satisfies

$$
\left\|Y_{0}\right\|_{X}+\left\|Y_{0}\right\|_{X_{\alpha}}+\left\|Y_{0}\right\|_{L_{1} \times L_{1}}+\left|\gamma_{0}\right| \leq \delta_{\alpha}^{0}, \text { for small } \delta_{\alpha}^{0}>0 .
$$

Noting that the semigroups $\mathrm{e}^{L_{1} t}$ and $\mathrm{e}^{L_{2} t}$ satisfy estimate (4.25) and

$$
\left\|\mathrm{e}^{L_{2} t}\right\|_{L_{1}(\mathbb{R}) \rightarrow L_{1}(\mathbb{R})} \leq 1 \text {, and }\left\|\mathrm{e}^{L_{2} t}\right\|_{L_{1}(\mathbb{R}) \rightarrow L_{\infty}(\mathbb{R})} \leq \frac{1}{\sqrt{t}}, \forall t>0,
$$


thus

$$
\left\|\mathrm{e}^{L_{2} t} y_{2}^{0}\right\|_{L_{\infty}(\mathbb{R})} \leq \frac{2}{\sqrt{1+t}}\left(\left\|y_{2}^{0}\right\|_{L_{1}(\mathbb{R})}+\left\|y_{2}^{0}\right\|_{L_{\infty}(\mathbb{R})}\right), \quad \forall t \geq 0 .
$$

By virtue of (4.16) (4.19), (4.27) and (4.28) for all $t \geq 0$, as in the proof of (4.33) we can similarly prove that for small $\sigma_{\alpha}>0$ under the assumption of (4.40) with small enough $\delta_{\alpha}^{0}>0$, it holds that

$$
\left\|y_{1}(\cdot, t)\right\|_{L_{1}(\mathbb{R})} \leq C_{2} \mathrm{e}^{-\frac{\sigma_{\alpha} t}{2}}\left(\left\|y_{1}^{0}\right\|_{L_{1}(\mathbb{R})}+\left\|Y_{0}\right\|_{X_{\alpha}}\right), \quad \forall t \geq 0 .
$$

By (4.34), (4.35) and (4.42), we have

$$
\begin{aligned}
& \left\|y_{2}(\cdot, t)\right\|_{L_{\infty}(\mathbb{R})} \leq\left\|\mathrm{e}^{L_{2} t} y_{2}^{0}\right\|_{L_{\infty}(\mathbb{R})}+C_{0} \int_{0}^{t}\left\|\mathrm{e}^{L_{2}(t-s)}\left(\tilde{H}_{2}(\cdot, s, Y, \gamma)+g_{2}(\cdot, s, \gamma)\right)\right\|_{L_{\infty}(\mathbb{R})} \mathrm{d} s \\
& \leq \frac{2}{\sqrt{1+t}}\left(\left\|y_{2}^{0}\right\|_{L_{1}(\mathbb{R})}+\left\|y_{2}^{0}\right\|_{L_{\infty}(\mathbb{R})}\right) \\
& \quad+C \int_{0}^{t} \frac{1}{\sqrt{1+t-s}}\left(\left\|y_{1}(\cdot, s)\right\|_{L_{\infty}(\mathbb{R})}+\left\|y_{1}(\cdot, s)\right\|_{L_{1}(\mathbb{R})}+\left\|\widehat{y}_{2}(\cdot, s)\right\|_{L_{\infty}(\mathbb{R})}+\left|\gamma^{\prime}(s)\right|\right) \mathrm{d} s, \forall t \geq 0 ;
\end{aligned}
$$

which with (4.16), (4.33), (4.40) and (4.43) further implies

$$
\left\|y_{2}(\cdot, t)\right\|_{L_{\infty}(\mathbb{R})} \leq \frac{2}{\sqrt{1+t}} \delta_{\alpha}^{0}+C \delta_{\alpha}^{0} \int_{0}^{t} \frac{1}{\sqrt{1+t-s}} \mathrm{e}^{-\frac{\sigma_{\alpha}}{2} s} \mathrm{~d} s \leq \frac{C^{*} \delta_{\alpha}^{0}}{\sqrt{1+t}}, \quad \forall t \geq 0 .
$$

By (4.16), (4.34), (4.35), (4.41) and (4.43), we can similarly prove that

$$
\begin{aligned}
& \left\|y_{2}(\cdot, t)\right\|_{L_{1}(\mathbb{R})} \leq\left\|y_{2}^{0}\right\|_{L_{1}(\mathbb{R})}+\int_{0}^{t}\left(\left\|\tilde{H}_{2}(\cdot, s, Y, \gamma(s))\right\|_{L_{1}(\mathbb{R})}+\left\|G_{2}(\cdot, s, \gamma(s))\right\|_{L_{1}(\mathbb{R})}\right) \mathrm{d} s \\
& \leq \delta_{\alpha}^{0}+C \delta_{\alpha}^{0} \int_{0}^{t} \mathrm{e}^{-\frac{\sigma_{\alpha}}{2} s} \mathrm{~d} s \leq C^{*} \delta_{\alpha}^{0} .
\end{aligned}
$$

Thus estimates (1.19) and (1.20) follow from (1.18) and (4.43)-(4.45). This completes the proof of Theorem 1.4.

Proof of Theorem 1.5. For the case (1.7) and $c>c_{*}^{0}$, by virtue of Theorem 3.9, Theorem 1.5 can be proved by similar argument as in the proof of Theorem 1.4 with $\gamma(t) \equiv 0$, and the proof can be much simpler. A similar proof or estimates can also be found in [21] for the case (1.10) but in different weighted spaces. Here we omit the details of the proof.

Acknowledgements. The authors are very grateful to the anonymous referees for their detailed comments and many useful suggestions which helped improve the exposition of the current paper. Yi Li is supported by Changjiang research grant at Xian Jiaotong University. Yaping Wu is supported by NSFC (11071172), Beijing NSF (1132003), Beijing Municipal Education Commission (KZ201310028030) and SRFDP (20101108110001).

\section{References}

[1] S. Ai, W. Huang. Traveling wave fronts in combustion and chemical reaction models. Proc. Royal Soc. Edin. 137A, (2007), 671-700.

[2] J. Alexander, R. Gardner, C. Jones. A topological invariant arising in the stability of traveling waves. J. Reine Angew. Math., 410 (1990), 167-212.

[3] N.J. Balmforth, R.V. Crastev, J.A. Malham. Unsteady fronts in an autocatalytic system. Proc. R. Soc. Lond. A, 455 (1999), 1401-1433.

[4] J.W. Bebernes, C.-M. Li, Y. Li. Traveling fronts in cylinders and their stability. Rocky Mountain J. Math., 27 (1997), 123-150.

[5] H. Berestycki, L. Nirenberg. Traveling fronts in cylinders. Ann. Institute. Henri Poincare, Analysis non lineaire, 9 (1992), 497-572. 
[6] J. Billingham, D.J. Needham. A note on the properties of a family of traveling wave solutions arising in cubic autocatalysis. Dyn. Stab. Syst., 6 (1991), 33-49.

[7] M. Bramson. Convergence of solutions of the Kolmogorov equations to travelling waves. Mem. Amer. Math. Soc., 44(1983).

[8] J. Carr. Application of centre manifold theory. Applied Mathematical Sciences, Vol.35, Springer-Verlag, New York, 1981.

[9] X.F. Chen, Y.W. Qi. Sharp estimates on minimum travelling wave speed of reacton-diffusion systems modelling auto-catalyst. SIAM J.Math. Anal., 39 (2007), 437-448.

[10] X.F. Chen, Y.W. Qi. Travelling waves of auto-catalytic chemical reaction of general order- An elliptic approach. J. Differential Equations, 246 (2009), 3038-3057.

[11] S. Focant, Th. Galley. Existence and stability of propagating fronts for an autocatalytic reactio-diffuion systems. Physica D, 120 (1998), 346-368.

[12] A. Ghazaryan, Y. Latushkin, S. Schecter. Stability of traveling waves for a class of reaction-diffusion systems that arise in chemical reaction models. SIAM J. Math. Anal., 42 (2010), 2434-2472.

[13] F. Hamel, L. Roques. Fast propagation for KPP equations with slowly decaying initial conditions. J. Differential Equations, 249 (2010), 1726-1745.

[14] D. Henry. Geometric theory of semilinear parabolic equations. Lecture Notes in Mathematics 840, Springer-Verlag, New York, 1981.

[15] Y. Hosono. Propagation speeds of traveling fronts for higher order autocatalytic reaction-diffusion systems. Japan J. Indust. Appl. Math., 24 (2007), 79-104.

[16] X. Hou, Y. Li. Local stability of traveling wave solutions of nonlinear reaction-diffusion equations, Discrete Contin. Dyn. Syst., 15 (2006), 681-701.

[17] T. Kato. Peturbation theory for linear operators. corrected printing of the second edition, Springer-Verlag, Berlin, 1980.

[18] K. Kirchgässner, G. Raugel. Stability of fronts for a KPP-System II - the critical case -. J. Differential Equations, 146 (1998), 399-456.

[19] M. Marion. Qualitative properties of a nonlinear system for laminar flames without ignition temerature. Nonl. Anal. TMA, 9 (1998), 1269-1292.

[20] M.J. Metcalf, J.H. Merkin, S.K. Scott. Oscillating wave fronts in isothermal chemical systems with arbitary powers of autocatalysis. Proc. R. Soc. Lond. B, 447 (1994), 155-174.

[21] Y. Li, Y. Wu. Stability of traveling front solutions with algebraic spatial decay for some auto-catalytic chemical reaction systems. SIAM J. Math. Anal., 44 (2012), 1474-1521.

[22] A. de Pablo, J. L. Vazquez. Travelling wave behavior for a Porous-Fisher equations. Euro. J. Applied Mathematics, 9 (1998), 285-304.

[23] R.L. Pego , M.I. Weinstein. Eigenvalues, and instability of solitary waves. Philos. Trans. Roy. Soc. London Ser. A, 340 (1992), 47-94.

[24] F. Rothe. Covergence to travelling fronts in semilinear parabolic equations. Proc. Roy. Soc. Edinburgh, Sect A, 80 (1978), 213-234.

[25] D.H. Sattinger. On the stability of waves of nonlinear parabolic systems. Advances in Math., 22 (1976), $312-255$.

[26] J.A. Sherratt, B.P. Marchant. Algebraic decay and variable speeds in wavefront solutions of a scalar reaction-diffusion equation. IMA J. Appl. Math., 56 (1996), 289-302.

[27] H. Takase, B.D. Sleeman. Travelling-wave solutions to monostable reaction-diffusion systems of mixed monotone type. Proc. R. Soc. Lond. A, 455 (1999), 1561-1598.

[28] K. Uchiyama. The behaviour of solutions of some semilinear diffusion equations for large time. J. Math. Kyoto. Univ., 18 (1978), 453-508.

[29] Y. Wu, Y.X. Wu. Asymptotic behavior of solution to degeneate Fisher equations with algebraic decaying initial values. peprint.

[30] Y. Wu, X. Xing. The stability of travelling waves with critical speeds for p-degree Fisher-type equation. Discrete Contin. Dyn. Syst. A, 20 (2008), 1123-1139.

[31] Y. Wu, X. Xing. The stability of travelling fronts for general scalar viscous balance law. J. Math. Anal. Appl., 305 (2005), 698-711.

[32] Y. Wu, X. Xing, Q. Ye. Stability of traveling waves with algebraic decay for $n$-degree Fisher-type equations. Discrete Contin. Dyn. Syst. A, 16 (2006), 47-66. 